\title{
Which is better for mothers and babies: fresh or frozen-thawed blastocyst transfer?
}

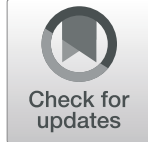

Meiling Yang ${ }^{1,2}$, Li Lin ${ }^{1,3}$, Chunli Sha ${ }^{1,3}$, Taoqiong Li ${ }^{1,3}$, Wujiang Gao ${ }^{1,3}$, Lu Chen ${ }^{1,3}$, Ying Wu ${ }^{1,3}$, Yanping Ma $a^{1,3}$ and Xiaolan Zhu ${ }^{1,3^{*}}$

\begin{abstract}
Background: In recent years, there have been many reports on the pregnancy outcomes of fresh blastocyst transfer (BT) and frozen-thawed BT, but the conclusions are controversial and incomplete. To compare the pregnancy outcomes, maternal complications and neonatal outcomes of fresh and frozen-thawed BT in the context of in vitro fertilization or intracytoplasmic sperm injection (IVF/ICSI) cycles, we conducted a meta-analysis.

Methods: A meta-analysis was conducted by searching the PubMed, Embase, and Cochrane Library databases through May 2020. Data were extracted independently by two authors.

Results: Fifty-four studies, including 12 randomized controlled trials (RCTs), met the inclusion criteria. Fresh BT was associated with a lower implantation rate, pregnancy rate, ongoing pregnancy rate, and clinical pregnancy rate and higher ectopic pregnancy rate than frozen-thawed BT according to the results of the RCTs. The risks of moderate or severe ovarian hyperstimulation syndrome, placental abruption, placenta previa and preterm delivery were higher for fresh BT than for frozen-thawed BT. The risk of pregnancy-induced hypertension and pre-eclampsia was lower for fresh BT; however, no significant differences in risks for gestational diabetes mellitus and preterm rupture of membrane were found between the two groups. Compared with frozen-thawed BT, fresh BT appears to be associated with small for gestational age and low birth weight. No differences in the incidences of neonatal mortality or neonatal malformation were observed between fresh and frozen-thawed BT.
\end{abstract}

Conclusions: At present there is an overall slight preponderance of risks in fresh cycles against frozen, however individualization is required and current knowledge does not permit to address a defintive response.

Keywords: Fresh blastocyst transfer, Frozen-thawed blastocyst transfer, Pregnancy outcome, Maternal complications, Neonatal outcomes

\footnotetext{
* Correspondence: zx12517@163.com

${ }^{1}$ Reproductive Medicine Center, The Fourth Affiliated Hospital of Jiangsu University (Zhenjiang Maternal and Child Health Hospital), 20 Zhengdong Road, Zhenjiang, Jiangsu 212001, People's Republic of China

${ }^{3}$ Reproductive Sciences Institute, Jiangsu University, Zhenjiang 212001, Jiangsu, China

Full list of author information is available at the end of the article
}

(c) The Author(s). 2020 Open Access This article is licensed under a Creative Commons Attribution 4.0 International License, which permits use, sharing, adaptation, distribution and reproduction in any medium or format, as long as you give appropriate credit to the original author(s) and the source, provide a link to the Creative Commons licence, and indicate if changes were made. The images or other third party material in this article are included in the article's Creative Commons licence, unless indicated otherwise in a credit line to the material. If material is not included in the article's Creative Commons licence and your intended use is not permitted by statutory regulation or exceeds the permitted use, you will need to obtain permission directly from the copyright holder. To view a copy of this licence, visit http://creativecommons.org/licenses/by/4.0/. The Creative Commons Public Domain Dedication waiver (http://creativecommons.org/publicdomain/zero/1.0/) applies to the data made available in this article, unless otherwise stated in a credit line to the data. 


\section{Background}

As cryopreservation technology has developed during the past few decades, the proportion of individual undergoing frozen blastocyst transfer (BT) has increased [1]. There have been concerns about the impact of cryopreservation on pregnancy outcomes, maternal complications and the health of the infant [2]. A few studies have compared pregnancy outcomes following fresh BT and cryopreserved-thawed BT in patients undergoing in vitro fertilization or intracytoplasmic sperm injection (IVF/ICSI) cycles [3-5]. However, the findings are controversial. A recent meta-analysis supported the hypothesis that single cryopreserved BT might not be a better choice than single fresh BT for patients undergoing IVF/ ICSI [3]. However, another systematic review and metaanalysis suggested that pregnancy outcomes may be improved when frozen-thawed BT is performed [4].

With regard to maternal complications, Maheshwari et al. showed that frozen-thawed BT was associated with lower risks of postpartum haemorrhage $(\mathrm{PH})$, placental abruption (PA), placenta previa (PP) and preterm delivery (PTD) than fresh BT and that the pregnancies arising from frozen-thawed BT seemed to have lower risks of maternal complications [2]. Shavit et al. reached the opposite conclusion that frozen-thawed BT may contribute to an increased risk of maternal complications such as preeclampsia and gestational diabetes mellitus (GDM) [6]. The latest randomized controlled trial reported that the incidence of preeclampsia was higher after frozenthawed BT than after fresh BT, and the risk of moderate or severe ovarian hyperstimulation syndrome (OHSS) was similar in both groups [7].

Regarding the neonatal outcomes, an early review demonstrated that there were no significant differences in incidences of perinatal death or low birth weight of infants between fresh BT and frozen-thawed BT [8]. However, another review suggested that the incidences of small for gestational age (SGA), low birth weight (LBW), and perinatal mortality were lower in women who received frozen thawed BT [2].

There is growing concern regarding whether children born after frozen thawed BT have higher risks of congenital malformations than those born after fresh BT via IVF/ICSI. A register-based cohort study suggested that the risk for congenital malformation of the children born after frozen thawed BT was not higher than that of children born after fresh BT; in addition, no increased risks regarding the affected organ system were found between the two groups [9].

Due to limited sample sizes, past meta-analysis conclusions are controversial. With the emergence of new reports, there is an urgent need to perform a meta-analysis to compare the outcomes following fresh BT and frozenthawed BT to provide guidance for clinical practice. The purpose of this meta-analysis was to examine pregnancy outcomes, maternal complications and neonatal malformations after frozen thawed BT versus those after fresh BT in an IVF/ICSI cycle and assess whether frozen thawed $\mathrm{BT}$ is a better choice than fresh BT.

\section{Methods}

We followed the Preferred Reporting Items for Systematic Reviews and Meta-Analyses (PRISMA) guidelines when performing the systematic review and metaanalysis (Additional file 1).

\section{Eligibility criteria}

The articles included compared clinical outcomes between patients undergoing IVF/ICSI cycles with fresh or frozen BT. Studies were excluded if they were duplicate publications, literature reviews or meta-analyses or if they had insufficient data for extraction.

\section{Search strategy}

We searched the articles published in the PubMed, EMBASE and Cochrane Library databases through May 2020, using the following terms as key words: 'humans', 'embryo"', 'cryo"', 'frozen', 'vitrif"', 'freez"', and 'fresh'. A comprehensive MEDLINE search strategy is presented in Additional file 2.

\section{Study selection and data extraction}

Two researchers evaluated the selected studies and extracted the data independently. Any disagreement was resolved by discussion. First, we excluded the obviously ineligible literature by reading the title and abstract and then further read the full texts to identify the studies to be included. The data extracted mainly consisted of the first author, year of publication, study design, sample size, participants, intervention protocol, results, and other characteristics.

\section{Assessment of risk of Bias}

We assessed the risk of bias of the included studies using RevMan 5.3.0 software and the risk of bias assessment of the Cochrane Collaboration [10], which assesses the generation of sequence allocation, allocation concealment, blinding, and incomplete outcome data for each trial included in the review. Each study was evaluated as having a high, low, or unclear risk of bias for each item. Any disagreements between the two reviewers were resolved by discussion with the corresponding author. The results of the assessment of risk of bias are shown in Additional file 3. Publication bias was checked by funnel plot if at least ten studies reported that outcome. Subgroup analysis was done by the design, cryopreservation type, endometrial preparation type, high ovarian response and single BT. Sensitivity analysis was 
employed to see the effect of single study on the overall estimation. Beyond subgroup analysis, meta-regression for the included studies was conducted to identify factors for heterogeneity.

\section{Outcome measures}

The pregnancy outcomes were as follows: Implantation rate reflected the number of gestational sacs seen per embryo transferred. Pregnancy was identified according to increased serum hCG levels within 10 days after blastocyst transfer. Ongoing pregnancy was defined as pregnancy continuing beyond the 10th gestational week. Clinical pregnancy was considered the presence of a gestational sac with foetal heart activity, as assessed by ultrasound at 7 weeks of gestation. Miscarriage included any pregnancy that did not become an ongoing pregnancy. A multiple pregnancy was defined as a gestation with more than one foetus. The live birth rate was calculated as the number of birthing events per embryo transfer. We recorded the following maternal complications: GDM, pregnancy-induced hypertension $(\mathrm{PIH})$, pre-eclampsia (PE), $\mathrm{PTD}, \mathrm{PP}, \mathrm{PA}, \mathrm{PH}$, and preterm rupture of membrane (PROM). PTD was defined as a liveborn or stillborn infant with a gestational age at birth of at least 28 weeks but less than 37 weeks. Very preterm delivery (VPTD) was defined as a liveborn or stillborn infant with a gestational age at birth of at least 28 weeks but less than 32 weeks. The neonatal outcomes included gestational age at delivery, birth weight, stillbirth, perinatal mortality and neonatal mortality. Large for gestational age (LGA) was defined as a birth weight above the 90th percentile. SGA was defined as birth weight below the 10th percentile. Very small for gestational age (VSGA) was defined as weighing below the 3rd percentile. High birth weight (HBW) was defined as a weight of $>4000 \mathrm{~g}$ at birth. Very high birth weight (VHBW) was defined as a weight of $>4500 \mathrm{~g}$ at birth. Low birth weight (LBW) was defined as a weight of $<2500 \mathrm{~g}$ at birth. Very low birth weight baby (VLBW) was defined as a weight of $<1500 \mathrm{~g}$ at birth. We also analysed neonatal malformations (NM), including congenital anomalies, chromosomal aberrations, and different organ system malformations.
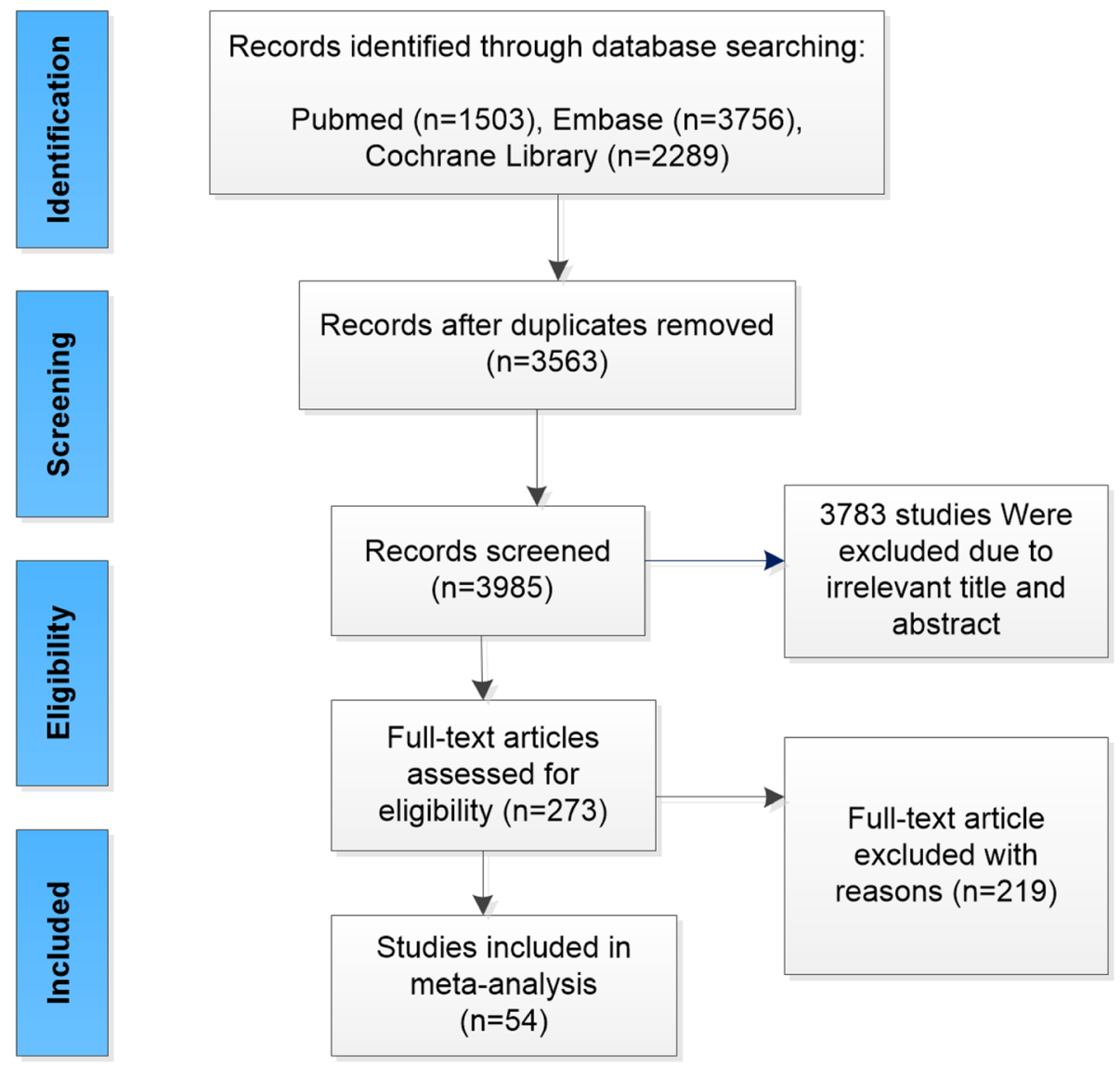

Fig. 1 Flowchart showing the study selection process for the meta-analysis 


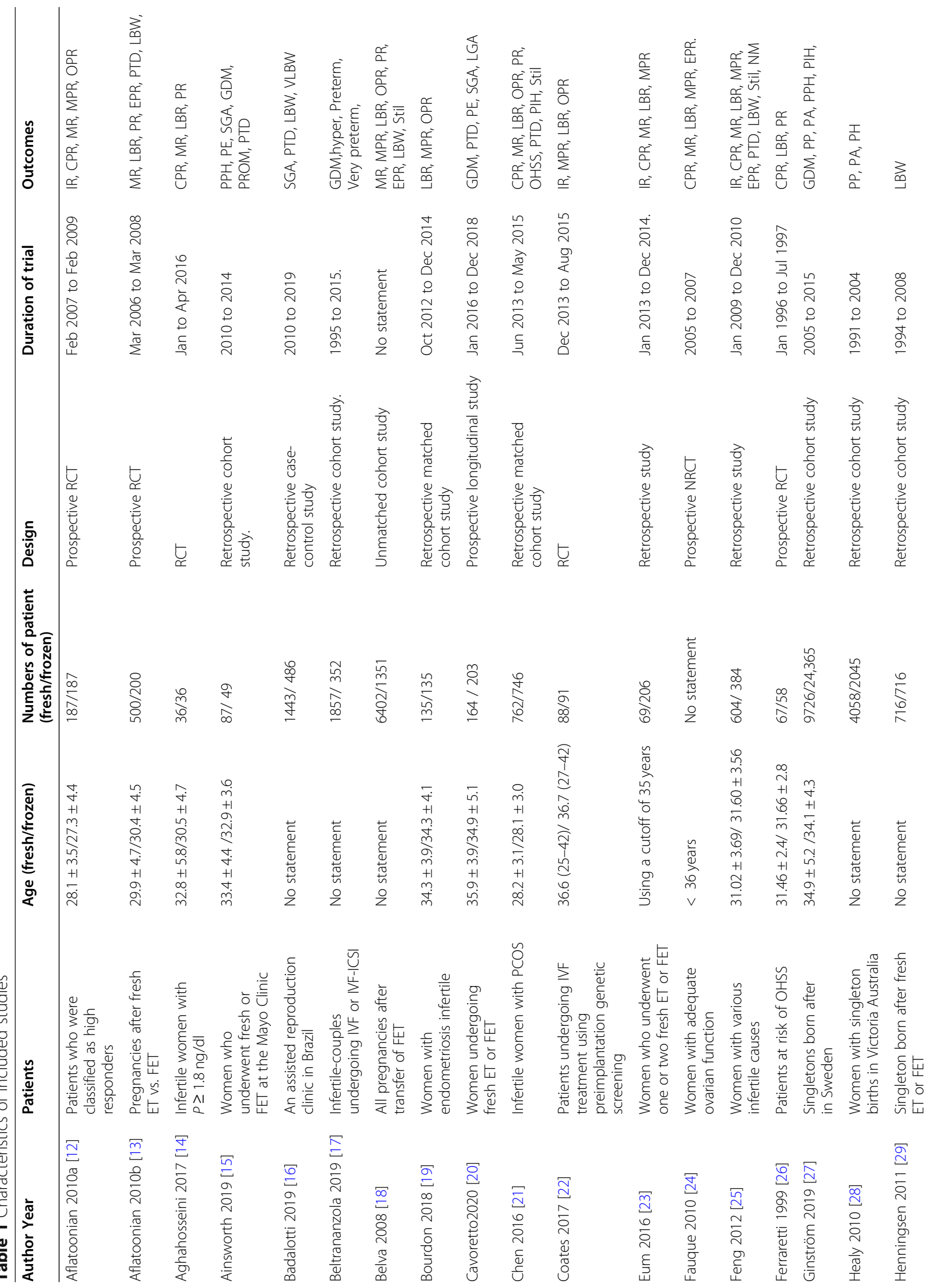




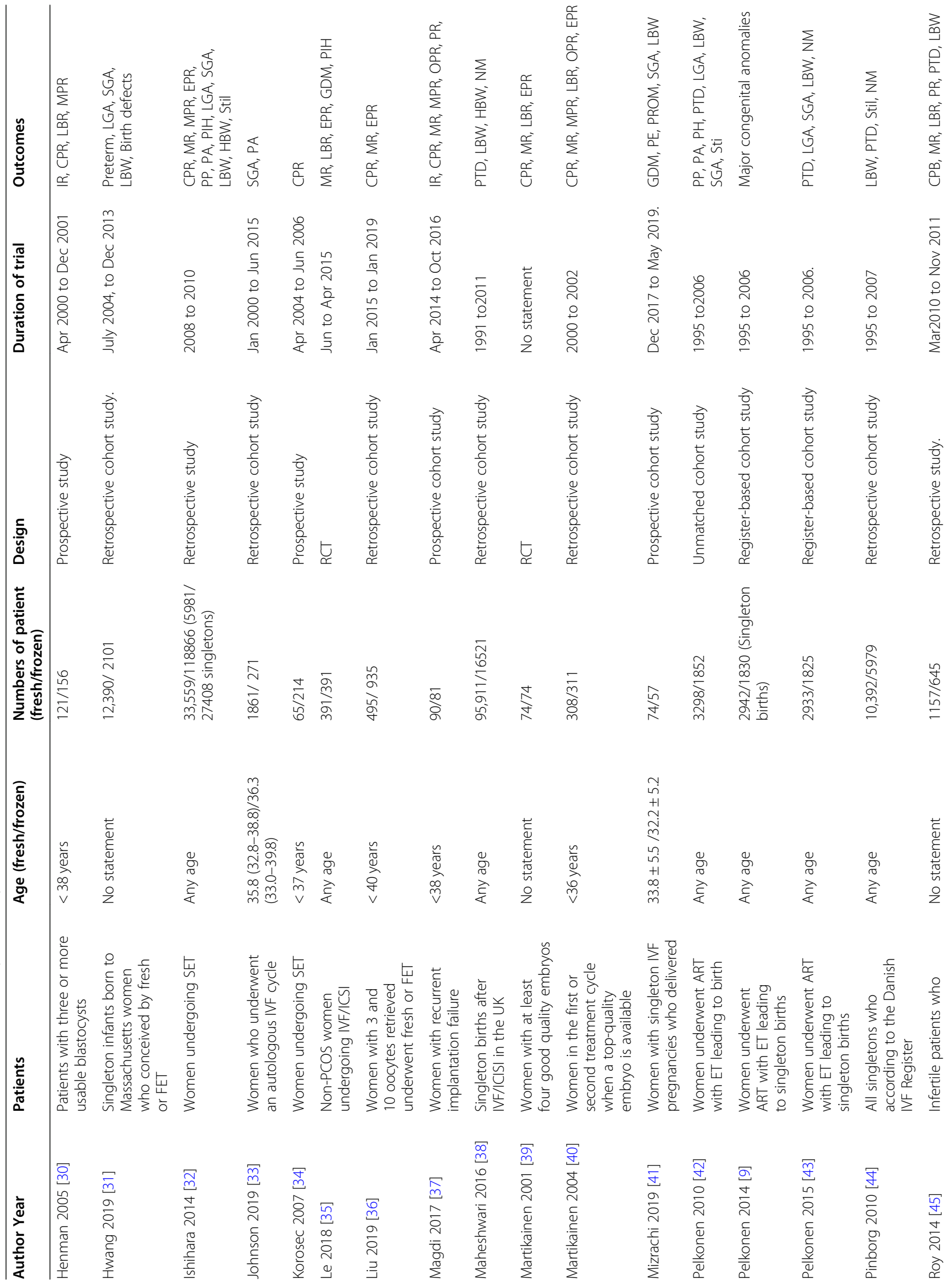




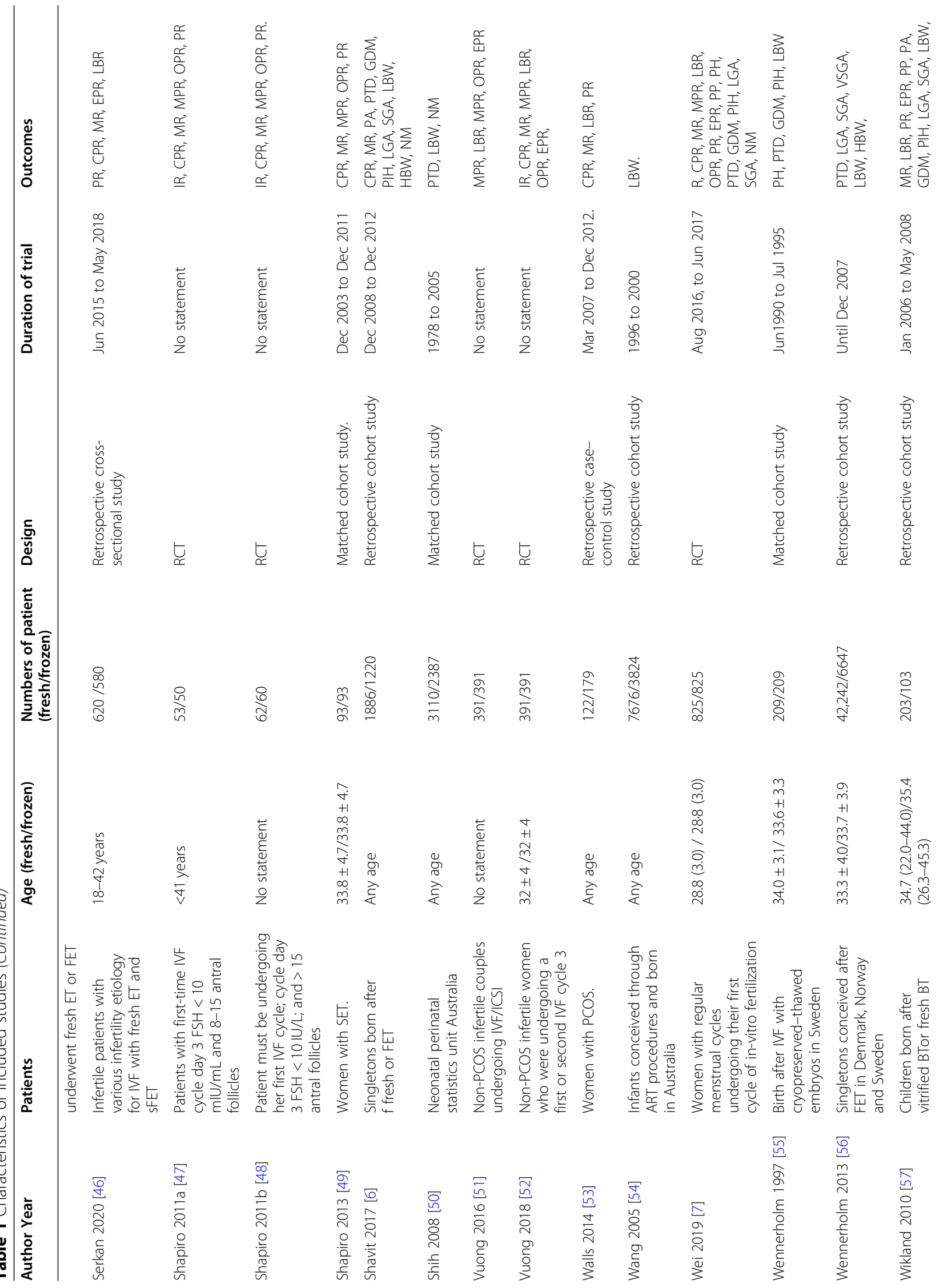




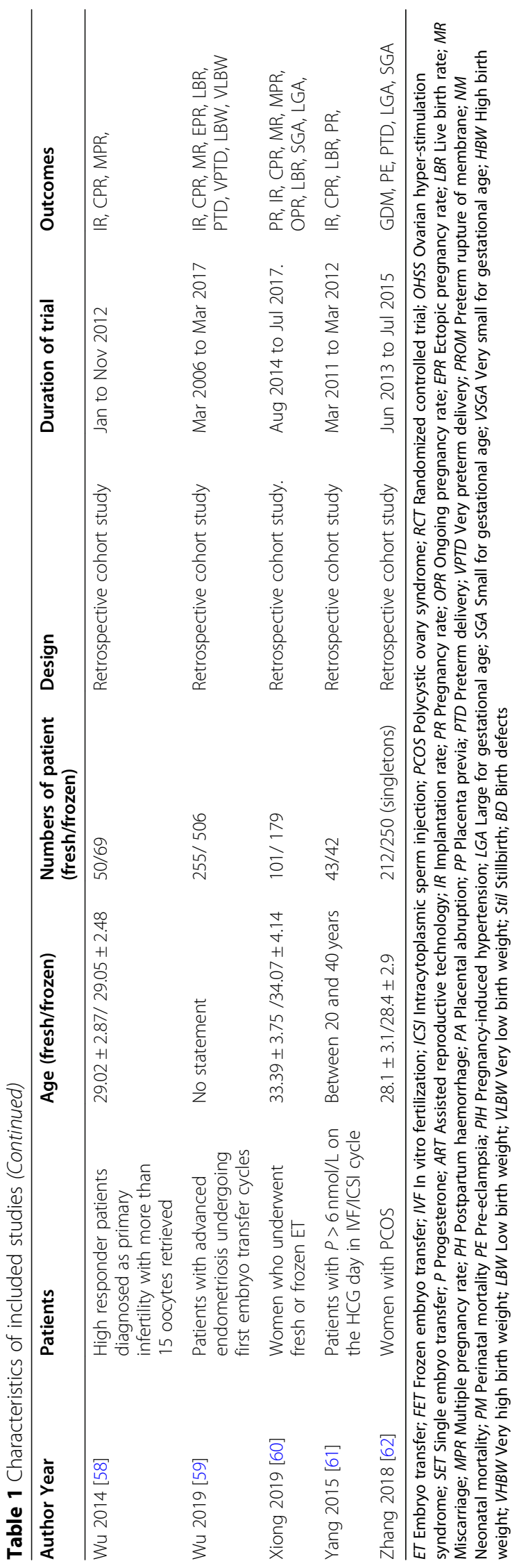




\section{Statistical analysis}

All statistical analyses were conducted using RevMan software. For the included studies, the results of dichotomous data for each of the studies eligible for metaanalysis were expressed as a risk ratio (RR) with the $95 \%$ confidence interval (CI). Although we analysed the data using both fixed effect models and random effect models, the results in the text are only from random effect models due to underlying heterogeneity in the studies. These results were combined for meta-analysis with the use of the Mantel/Haenszel model along with the random effects model. We assessed whether there was sufficient similarity between the eligible studies in regard to their design and clinical characteristics. Statistical heterogeneity was assessed with a chi-squared test and quantified with the $\mathrm{I}^{2}$ statistic. $\mathrm{I}^{2}>50 \%$ was considered to indicate marked heterogeneity [11]. $p<0.05$ was considered statistically significant.

\section{Results}

Study selection and characteristics

A total of 3985 available publications were identified in our search. Of these, 3783 were excluded after reading the title and the abstract. Finally, 54 articles, including 12 randomized controlled trials (RCTs) and 42 nonrandomized controlled trials (NRCTs), were considered eligible by one or both reviewers (Fig. 1). Table 1 provides the details of all included studies.

\section{Pregnancy outcomes}

A total 6 RCT reported that the implantation rate (IR) was lower in the fresh BT group than in the frozenthawed BT group (RR 0.79, 95\% CI 0.70-0.90, $P=$ 0.0004, heterogeneity: $I^{2}=65 \%$ ) (Fig. 2). Compared with frozen-thawed BT, fresh BT was associated with a lower pregnancy rate (PR) according to 6 RCTs (RR 0.83, 95\% CI 0.78-0.89, $P<0.00001$, heterogeneity: $I^{2}=0 \%$ ) (Fig. 3 ). Compared with women who underwent frozen-thawed $\mathrm{BT}$, women who underwent fresh BT showed a decreased OPR according to 6 RCTs (RR 0.80, 95\% CI 0.75-0.87, $P<0.00001$, heterogeneity: $\mathrm{I}^{2}=0 \%$ ) (Fig. 4). The clinical pregnancy rate (CPR) was lower in the fresh BT group than in the frozen-thawed BT group according to 7 RCTs (RR $0.86,95 \%$ CI $0.75-1.00, P=0.04$, heterogeneity: $I^{2}=56 \%$ ) (Fig. 5). Six RCTs showed that fresh $\mathrm{BT}$ resulted in a significantly higher ectopic pregnancy

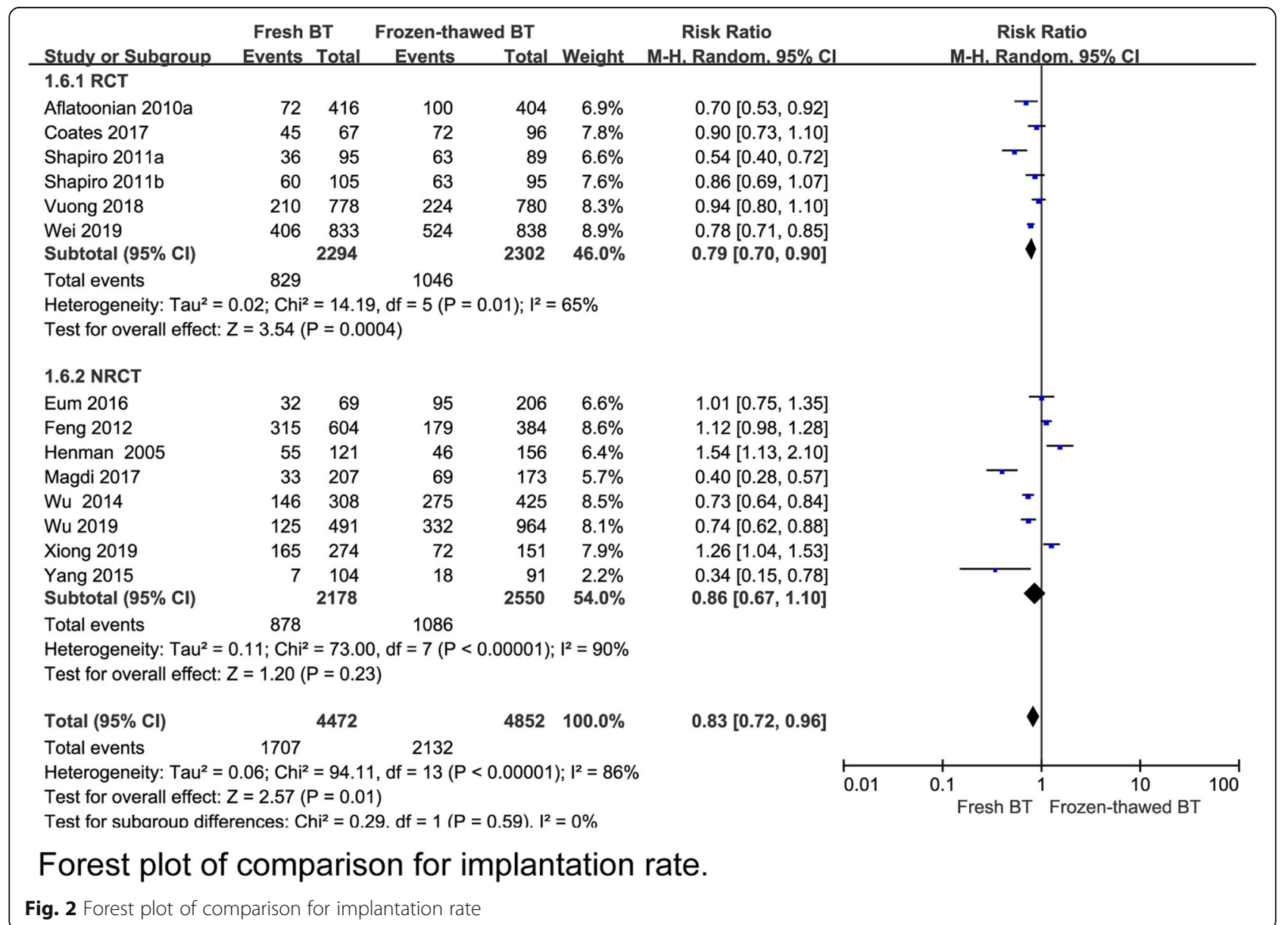




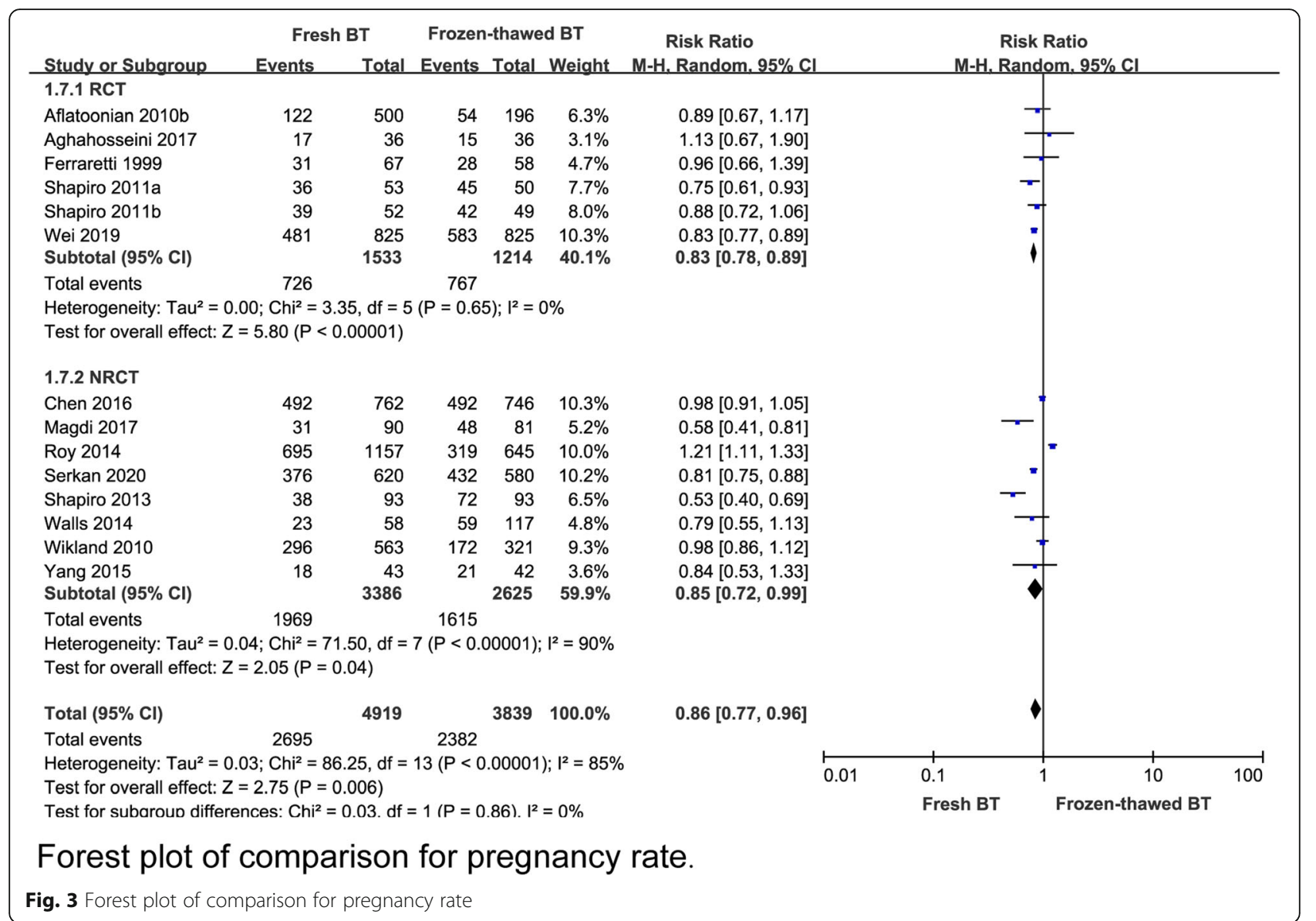

rate (EPR) than frozen-thawed $\mathrm{BT}$ (RR 1.96, 95\% CI 1.27-3.01, $P=0.002$, heterogeneity: $I^{2}=0 \%$ ) (Fig. 6). The above results indicate that frozen-thawed $\mathrm{BT}$ tends to result in higher IR, PR, OPR and CPR and lower EPR. According to the RCTs, the miscarriage rate (MR) (RR 0.86, 95\% CI 0.65-1.13, $P=0.27$, heterogeneity: $I^{2}=$ $37 \%$ ), MPR (RR 0.92, 95\% CI 0.70-1.21, $P=0.56$, heterogeneity: $I^{2}=30 \%$ ) and LBR (RR 0.92, 95\% CI 0.75-1.12, $P=0.41$, heterogeneity: $\left.I^{2}=87 \%\right)$ showed no statistically significant differences between fresh BT and frozenthawed BT (Appendix 4) (See Additional file 4).

In conclusion, our meta-analysis showed that fresh BT was associated with lower IR, PR, OPR, and CPR and higher EPR than frozen-thawed BT. There were no differences observed in the MR, MPR or LBR between fresh and frozen-thawed BT.

\section{Maternal complications}

To investigate whether fresh BT and frozen-thawed BT have different effects on maternal complications, we compared the incidence of $\mathrm{PIH}$, pre-eclampsia, OHSS, PTD, GDM, PP, PA, PH, and PROM between the two groups. Lower incidences of $\mathrm{PIH}$ and pre-eclampsia were observed for fresh BT than for frozen-thawed BT (RR
0.74, 95\% CI 0.63-0.87; $P=0.0002$, heterogeneity: $I^{2}=$ $57 \%$ ) (Fig. 7a). The incidence of GDM showed no statistically significant differences between the two groups (RR 0.94, 95\% CI 0.83-1.06; $P=0.32$, heterogeneity: $\mathrm{I}^{2}=$ 0\%) (Fig. 7b). Women who underwent fresh BT showed an increased risk of PA (RR 1.60, 95\% CI 1.26-2.04; $P=$ 0.0001, heterogeneity: $\mathrm{I}^{2}=0 \%$ ) (Fig. 8a). The risk of PP was higher for fresh BT than for frozen-thawed BT (RR 1.64, 95\% CI 1.23-2.19; $P=0.04$, heterogeneity: $I^{2}=60 \%$ ) (Fig. 8b). Compared with the frozen thawed BT group, the fresh group had higher risks of PTD (RR 1.24, 95\% CI 1.13-1.36, $P<0.00001$, heterogeneity: $I^{2}=73 \%$ ) (Fig. 8c) and VPTD (RR 1.27, 95\% CI 1.14-1.54, $P=0.037$, heterogeneity: $I^{2}=7 \%$ ) (Appendix 5) (See Additional file 4). The incidence of PH (RR 1.00, 95\% CI 0.57-1.74; $P=$ 0.99, heterogeneity: $I^{2}=93 \%$ ) and PROM (RR 1.05, 95\% CI 0.77-1.45; $P=0.74$, heterogeneity: $I^{2}=0 \%$ ) did not differ significantly between fresh BT groups and frozenthawed BT groups (Appendix 5) (See Additional file 4).

In summary, the risks of $\mathrm{PIH}$ and pre-eclampsia are lower for fresh BT than for frozen-thawed BT, while the risks of PA, PP and PTD for fresh BT are higher than those for frozen-thawed BT. However, the two groups had similar incidences of GDM, PROM and PH. 


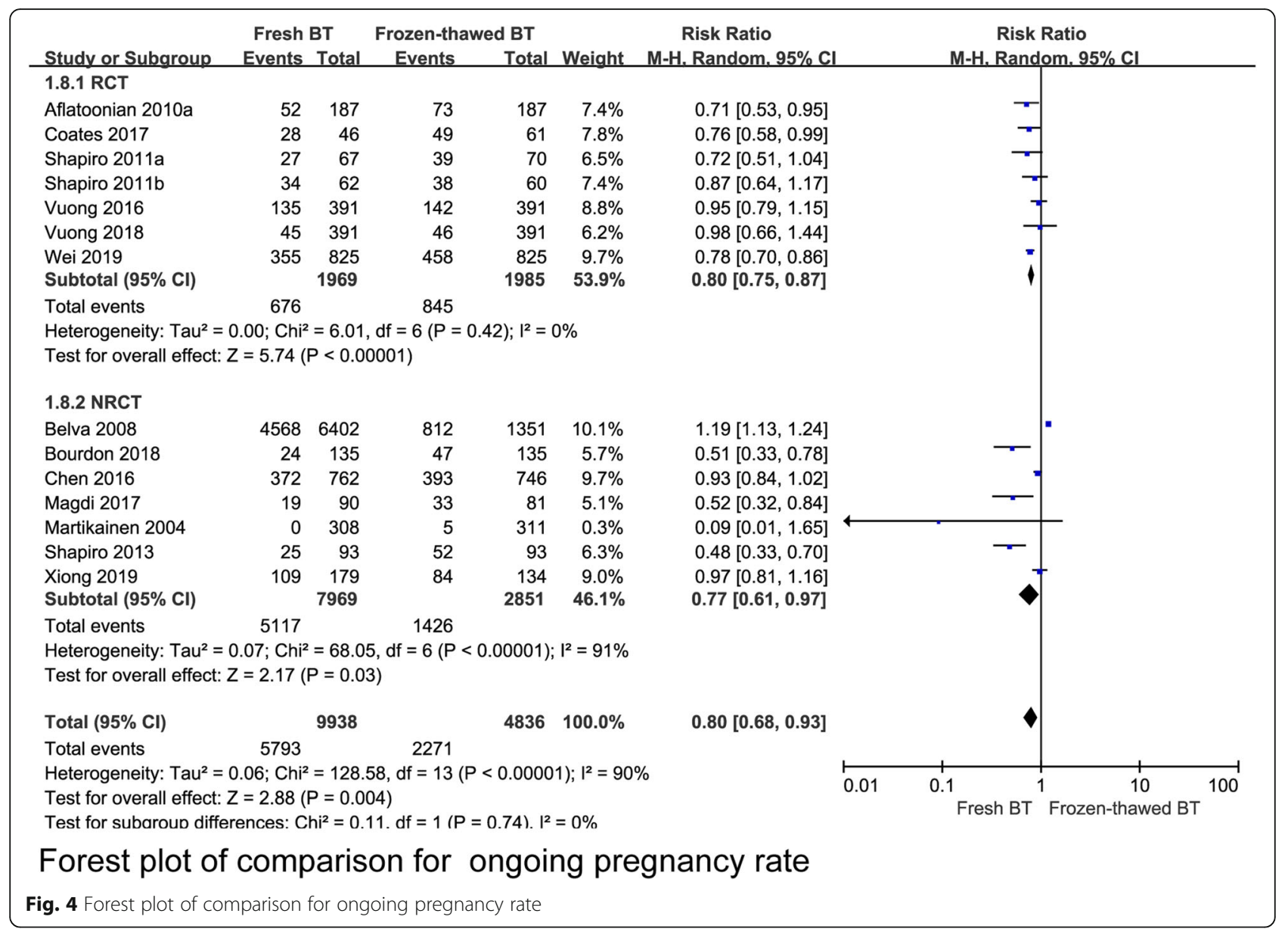

\section{Neonatal outcomes}

According to 12 studies, the LGA rate of the fresh BT group was lower (RR 0.62, 95\% CI 0.57-0.67, $P<$ 0.00001, heterogeneity: $I^{2}=39 \%$ ) (Fig. 9a). However, the SGA rate of the fresh BT group was higher than that of the frozen-thawed BT group according to the data from the 23 included studies (RR 1.24, 95\% CI 1.13-1.36, $P<0.00001$, heterogeneity: $I^{2}=73 \%$ ) (Fig. 9b). The RRs of HBW (RR 0.56 95\% CI $0.51-0.61$, $P<0.00001$, heterogeneity: $I^{2}=57 \%$ ) (Fig. 10a) and VHBW (RR 0.51, 95\% CI 0.36-0.71, P<0.0001, heterogeneity: $I^{2}=16 \%$ ) (Appendix 6) (See Additional file 4) in the fresh BT group showed an absolute decrease compared with those in the frozen thawed BT group. In contrast, the risks of LBW (RR 1.45, 95\% CI 1.341.57, $P<0.00001$, heterogeneity: $\mathrm{I}^{2}=60 \%$ ) (Fig. 10b) associated with fresh BT showed an absolute increase when compared with those associated with frozenthawed BT. No differences in VLBW (RR 0.97, 95\% CI $0.55-1.70, P=0.92$ heterogeneity: $I^{2}=96 \%$ ) were found (Appendix 6) (See Additional file 4). In addition, we investigated the differences in stillbirth, perinatal mortality and neonatal mortality between the two groups (Appendix 6) (See Additional file 4), and no significant differences were found.

In conclusion, fresh BT tends to lead to SGA and LBW, while frozen-thawed BT has the opposite effect. The stillbirth, perinatal mortality and neonatal mortality risks showed no statistically significant differences between the two groups.

\section{Neonatal malformations}

From the data we have summarized, the risks of congenital anomalies and chromosomal aberrations of newborns associated with fresh BT showed an absolute increase when compared with frozen-thawed BT (RR 1.09 95\% CI 1.02-1.17, $P=0.009$, heterogeneity: $\left.I^{2}=0 \%\right)$ (Fig. 11a). Further inspection of the risk of different organ system malformations in newborns, including malformations in the circulatory system (Fig. 11b), respiratory system (Fig. 11c), nervous system (Fig. 11d), gastrointestinal system, genitourinary system, eyes, ears, face, and musculoskeletal system (Appendix 7) (See Additional file 4), revealed no increased risk associated with frozen-thawed BT. The above data indicate that 


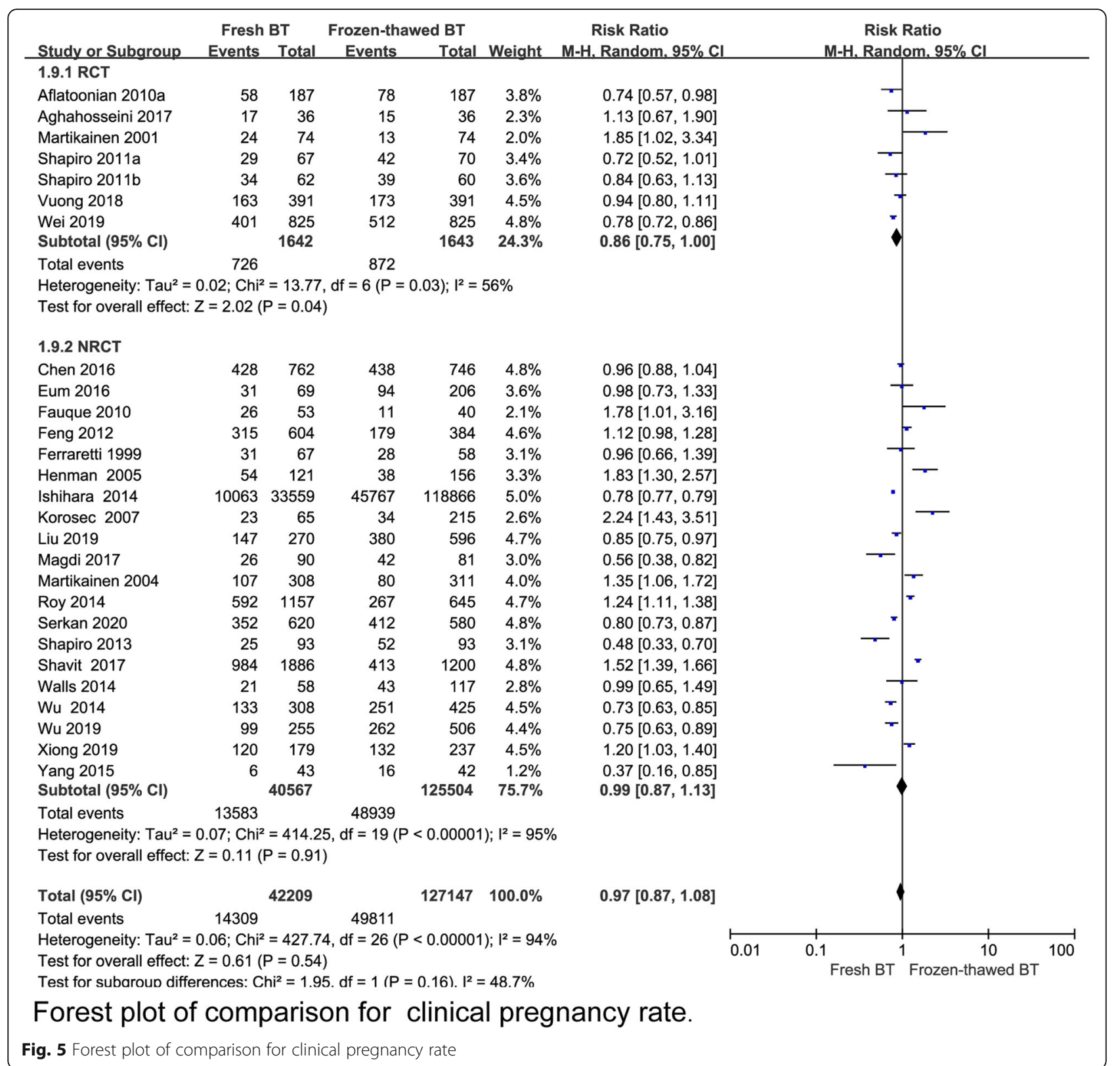

frozen-thawed BT is not a risk factor for neonatal malformations.

\section{Publication bias}

The publication bias could be assessed by using either a funnel plot subjectively and Eggers regression test objectively in Additional file 5. For this review, funnel plots showed a symmetrical distribution. Egger's regression test $p$-value on IR, PR, EPR, PIH \&PE, GDM, PTD, LGA and SGA was more than 0.05 and which indicated the absence of publication bias. Egger's tests yielding statistical evidence of publication bias at a $p$-value of OPR and CPR less than 0.05. In considering publication bias trim and fill meta-analysis was done. However, based on this analysis, no significant change was seen as compared with the main meta-analysis.

\section{Subgroup analysis}

Subgroup analysis was performed according to the design of the origin trials, cryopreservation method, endometrial preparation method (See Additional file 6). When both RCTs and NRCTs were included, we also reached a similar conclusion of higher IR, PR, OPR for frozen thawed BT, higher EPR for fresh BT, and no differences in CPR, LBR, MR or MPR. When using vitrification for blastocyst cryopreservation in frozen-thawed $\mathrm{BT}$, the trials revealed higher IR, OPR, LGA and lower EPR, SGA for frozen-thawed BT and no differences in 


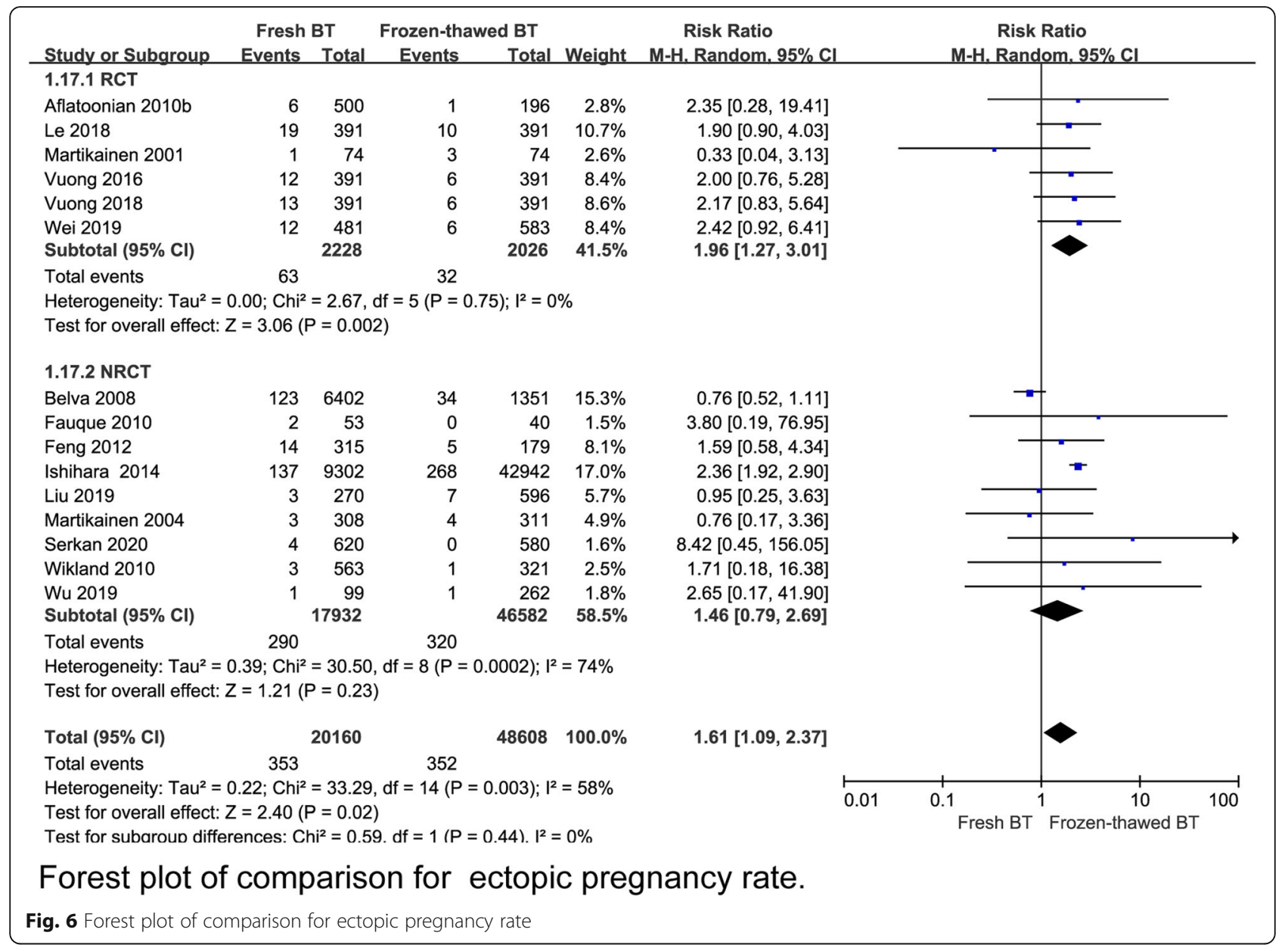

PR, CPR, LBR, MR, LBW or stillbirth. Trials adopting the slow-freezing protocol showed higher IR, PR, PIH, pre-eclampsia and LGA and lower LBR, SGA for frozen thawed BT, and no differences in OPR, CPR, MR, EPR, LBW or stillbirth were found. When using the artificial cycle of endometrial preparation in fresh $\mathrm{BT}$, the trials presented higher IR, PR, OPR, PIH and pre-eclampsia, LGA in. for frozen thawed BT and no differences in CPR, LBR, EPR, GDM, PA, PTD, SGA or LBW. The trials using the natural cycle showed higher IR, PR, CPR, and LGA, lower PA for frozen thawed BT and no differences in OPR, LBR, EPR, PIH, pre-eclampsia, GDM or PTD and lower SGA, LBW for frozen thawed BT. Moreover, there were a total of 4 studies analysing women with high ovarian response (See Additional file 7). Women with a high ovarian response who underwent frozen-thawed BT showed a higher IR, OPR and CPR and no difference in PR compared to women who underwent fresh BT. We also conducted a separate meta-analysis on single BT (See Additional file 8). Women who underwent single BT showed a higher OPR and lower EPR for frozen-thawed BT and no difference in IR, PR or CPR.

\section{Sensitivity analysis}

Sensitivity analysis was done to identify outlier studies. According to the analysis, when performing metaanalysis on PR, Belva 2008 exceeded the upper CI limit so this trial was excluded (Appendix 38) (Additional file 9). When performing the remaining metaanalysis, no influential studies were detected so all of the studies were included in the final analysis.

\section{Meta regression}

Beyond subgroup analysis, meta-regression for the included studies was conducted to identify factors for heterogeneity Meta-regression model in terms of natural cycle on IR, vitrification on PR, natural cycle on $C P R$, and slow-freezing on EPR was significant $(P<0.05)$ (Additional file 10). However, the meta-regression for the remaining results is not statistically significant.

\section{Discussion}

Great advances have been made in cryopreservation culture techniques for embryos since the success of the first pregnancy resulting from frozen-thawed embryo transfer (FET) in 1983 [63]. This technique has been applied as a 


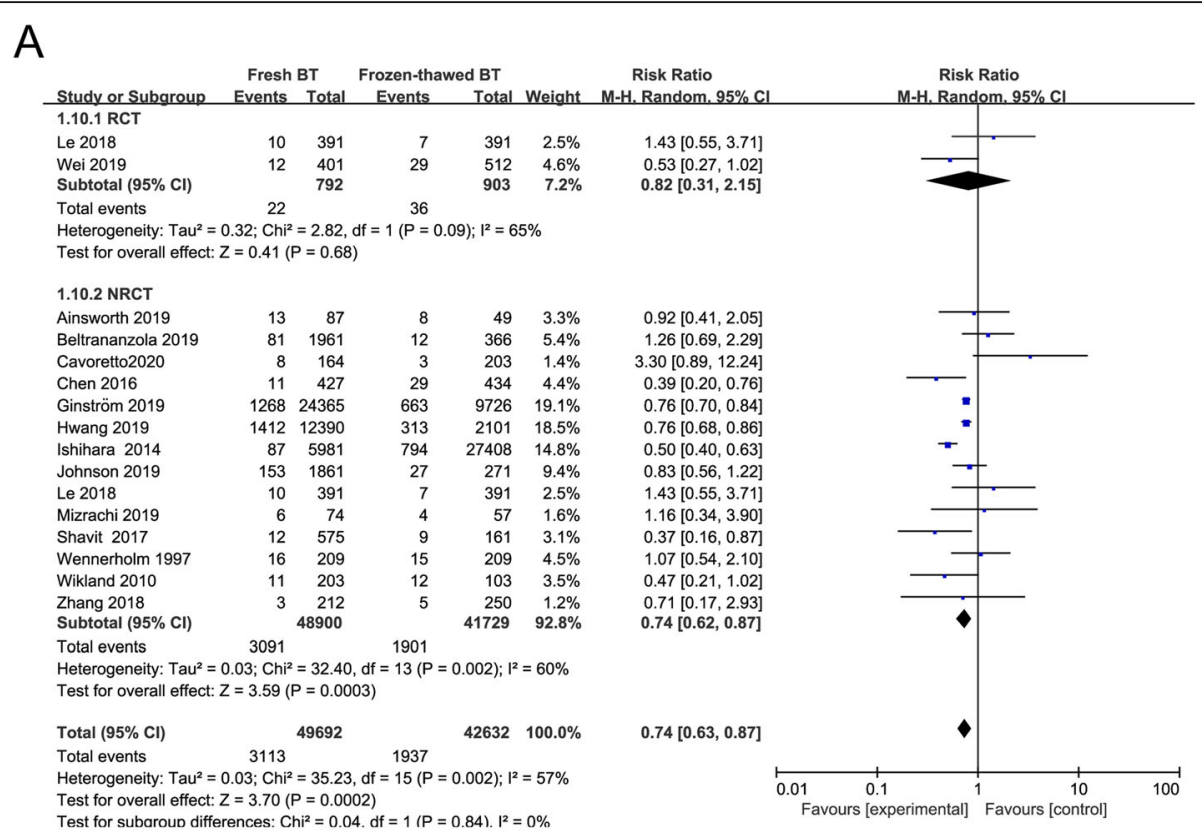

Forest plot of comparison for PIH and pre-eclampsia.

B

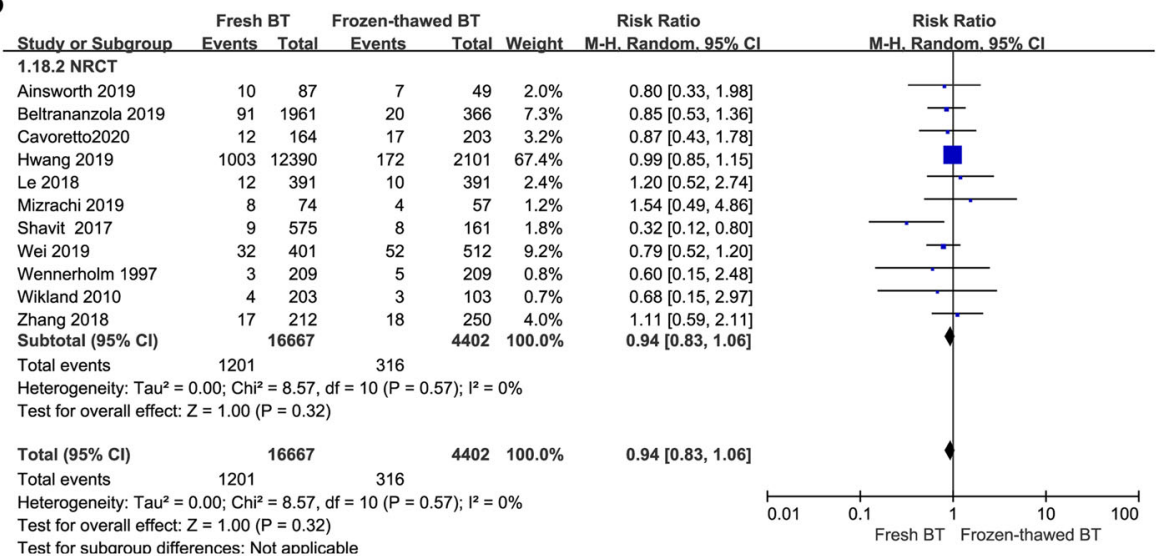

Forest plot of comparison for GDM

Fig. 7 Forest plot of comparison for (a) PIH and pre-eclampsia and (b) GDM

supplement to IVF and embryo transfer. FET has been accepted by every centre and has become an essential part of IVF/ICSI treatment. Therefore, the increased use of FET has increased the awareness of the safety of the technique [64]. This meta-analysis compared the outcomes of fresh BT and frozenthawed BT in individuals undergoing IVF/ICSI cycles, with comprehensive assessment of the pregnancy outcomes, maternal complications, neonatal outcomes and malformations. Our study showed that frozen BT was associated with increased IR, PR, OPR, CPR, PIH, pre-eclampsia, LGA and HBW. Fresh BT was associated with increased EPR, OHSS, PP, PA, PTB, SGA, LBW, congenital anomalies and chromosomal aberrations in newborns. There were no differences in CPR,
MR, MPR, LBR, PH or PROM between the two groups.

With respect to pregnancy outcomes, frozen-thawed BT was associated with higher IR, PR, OPR and CPR than fresh BT. There was no difference in MR, MPR, or LBR. Recently, Zeng et al. showed that there was no difference in IR, CPR, MR, or MPR, but an increased LBR was found for fresh BT compared with that of cryopreserved thawed BT [3]. Roque et al. showed that frozenthawed BT significantly improved CPR and OPR in patients undergoing IVF/ICSI cycles [4]. In general, there is more evidence that frozen-thawed BT is associated with better pregnancy outcomes than fresh BT. There may be several reasons, which are described as follows. Embryo implantation is one of the important steps for 
A

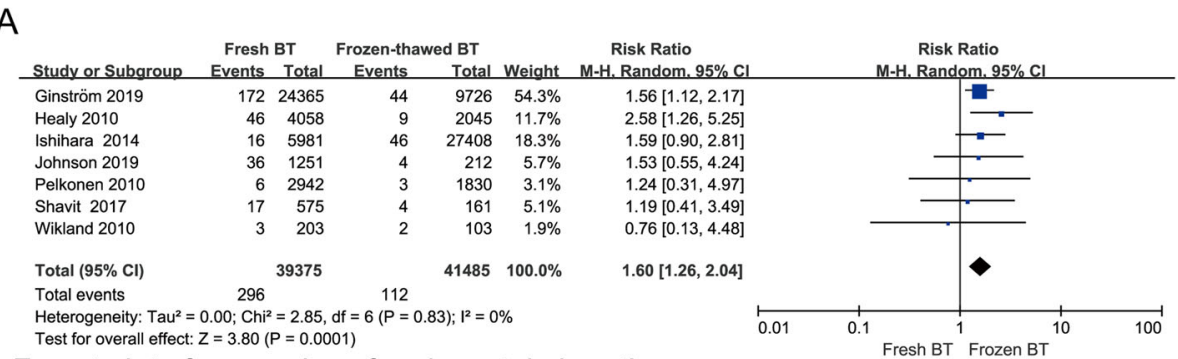

Forest plot of comparison for placental abruption

B

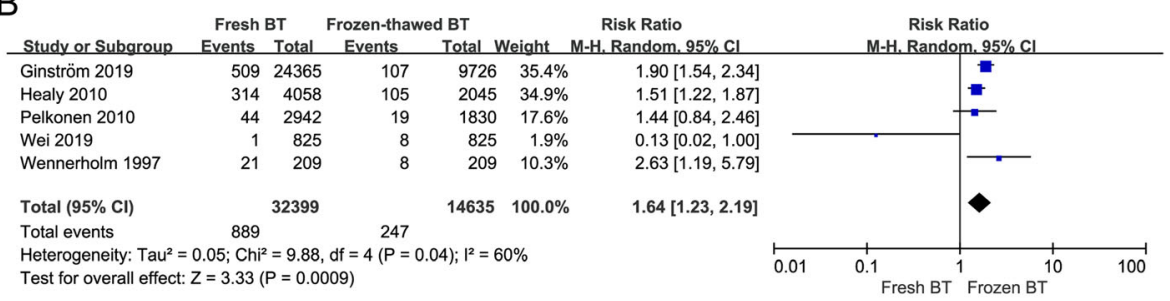

Forest plot of comparison for placenta previa

C

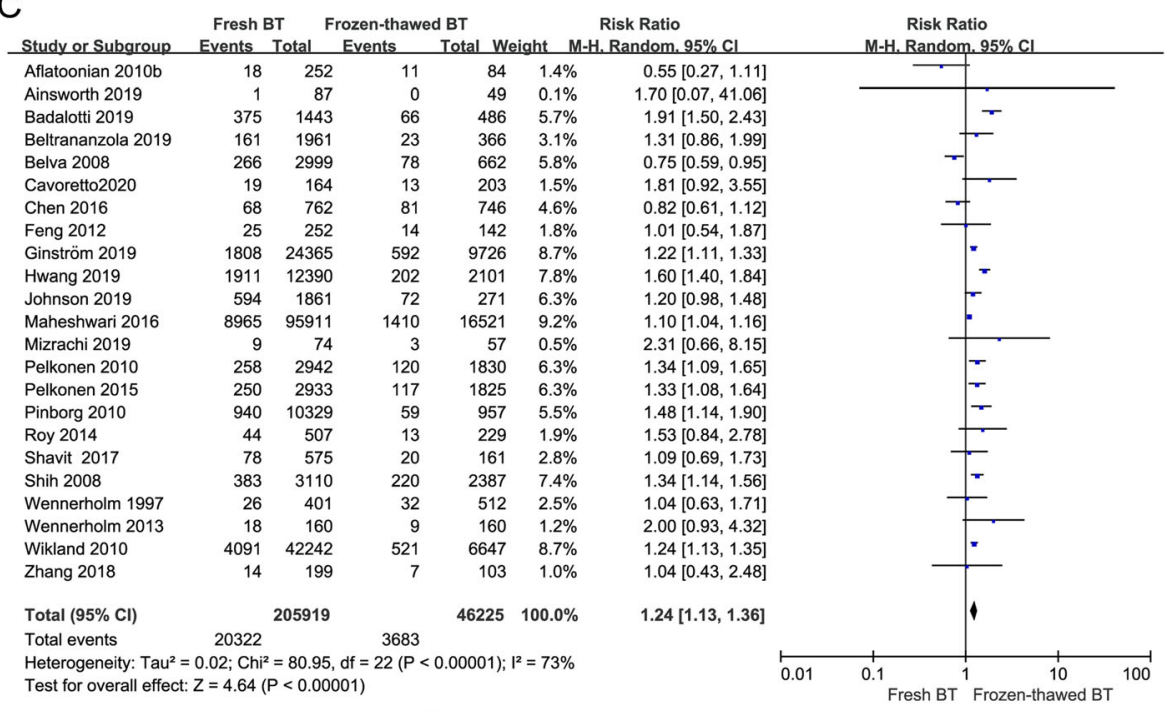

Forest plot of comparison for PTD

Fig. 8 Forest plot of comparison for (a) placental abruption, (b) placenta previa and (c) PTD

reproductive success, and implantation failure remains an unsolved problem in the field of IVF/ICSI. The primary cause of failure is the impairment of endometrial receptivity (ER), rather than the embryo itself being responsible for the failure [65]. A study suggested that impaired ER is more likely to occur in fresh ET cycles after ovarian stimulation than in FET cycles with artificial endometrial preparation. Impaired ER apparently accounted for most implantation failures in the fresh group [47]. Another explanation for the association of better pregnancy outcomes with frozen BT is that the physical effects of freezing and thawing embryos may filter out weaker embryos and allow only good quality embryos to survive, resulting in better foetal growth [50].
The incidence of EPR between the two groups varied in different studies. The inconsistent conclusions may be related to differences in the data included. A previous study reported that EPR was higher for frozen thawed BT [66]. However, our study suggested that frozen thawed BT was related to lower EPR, which is consistent with these studies $[67,68]$. The reason may be the high gonadotropin levels in ovarian stimulation cycles [68]. These findings also support the conclusions that a negative effect of ovarian stimulation on endometrial receptivity was reflected by increased EPR rates in the context of fresh BT.

From the perspective of maternal complications, our research demonstrated that the risks of PA, PP and PTD 


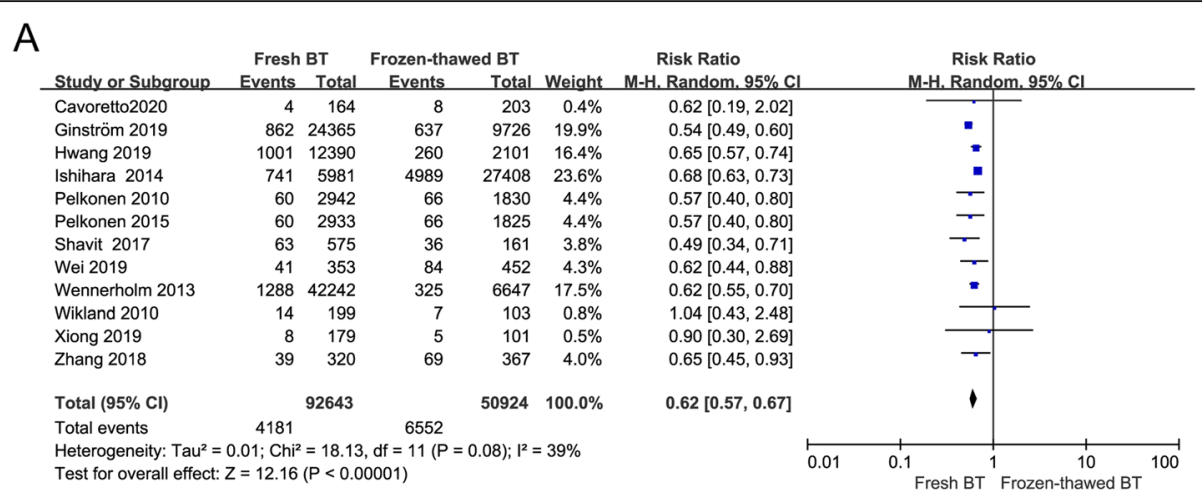

Forest plot of comparison for large for gestational age.

B

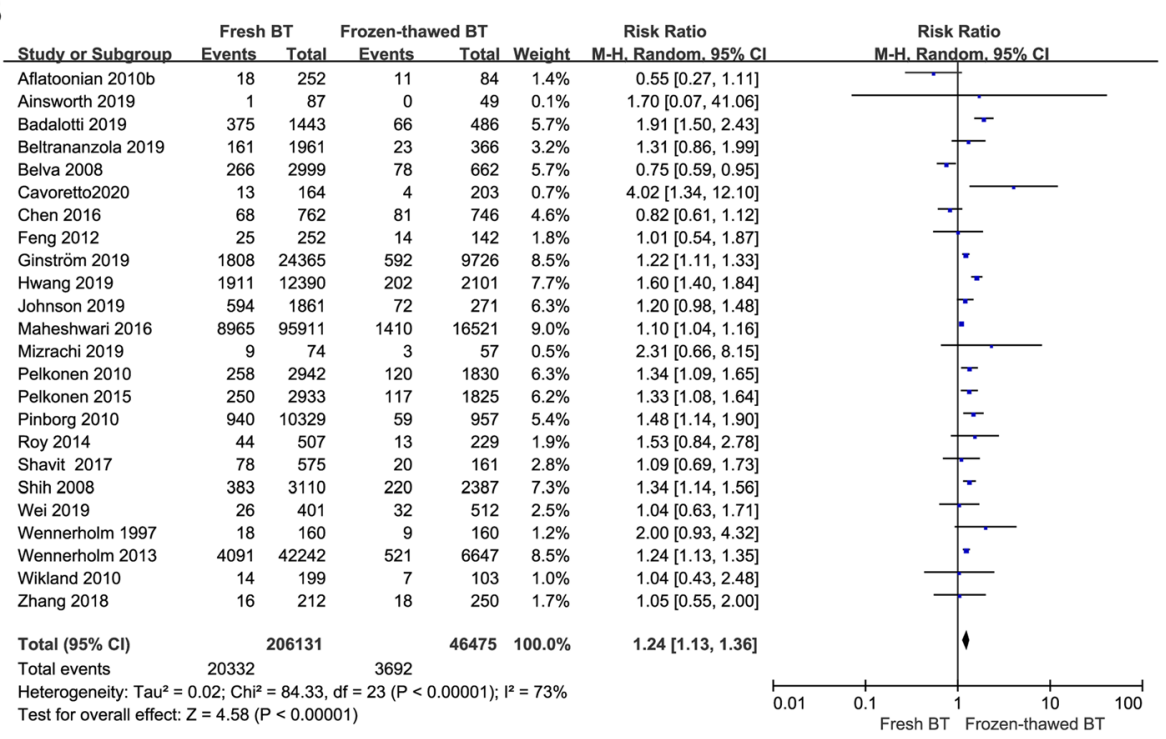

Forest plot of comparison for small for gestational age.

Fig. 9 Forest plot of comparison for (a) large for gestational age, (b) small for gestational age

are lower for frozen-thawed BT than for fresh BT. In contrast, the risks of $\mathrm{PIH}$ and preeclampsia associated with frozen-thawed BT are higher than those associated with fresh BT. Blazquez et al. suggested that FET pregnancies carry a higher risk of PIH and pre-eclampsia than fresh ET pregnancies, possibly indicating that the protocols currently used for endometrial reception of embryos have a deleterious effect on placentation, perhaps due to the long exposure to hormone replacement [69]. OHSS is an iatrogenic condition resulting from an excessive ovarian response to superovulation medication. According to a previous meta-analysis, no difference was found in OHSS between fresh BT and frozen-thawed BT [70]. However, the previous data were insufficient. In our studies, 4 trials involving freezing cycles triggered with HCG showed that the risk of OHSS was higher for fresh BT than for frozen-thawed BT. A few recent reviews demonstrated that singleton pregnancies after the transfer of frozen-thawed embryos were associated with lower risks of PTD and VPTD than those after fresh embryo transfer, which is in agreement with our research [2, 71-73]. A recent meta-analysis showed that spontaneous preterm birth (SPTB) is increased in individuals undergoing IVF/ICSI [74]. The subgroup sensitivity analysis excluding FET revealed a higher risk of SPTB than that in the general group, supporting the hypothesis of lower prematurity risk in pregnancies from FET [74]. A recent meta-analysis of cohort studies showed that it is possible that a shift toward FET will determine a reduction in iatrogenic preterm birth. However, the authors advocated caution and further prospective studies to avoid the risk of increasing other negative obstetrics outcomes [75].

In regard to neonatal outcomes, our study suggested that there were lower risks of SGA and LBW in singleton pregnancies after frozen-thawed BT than in those 


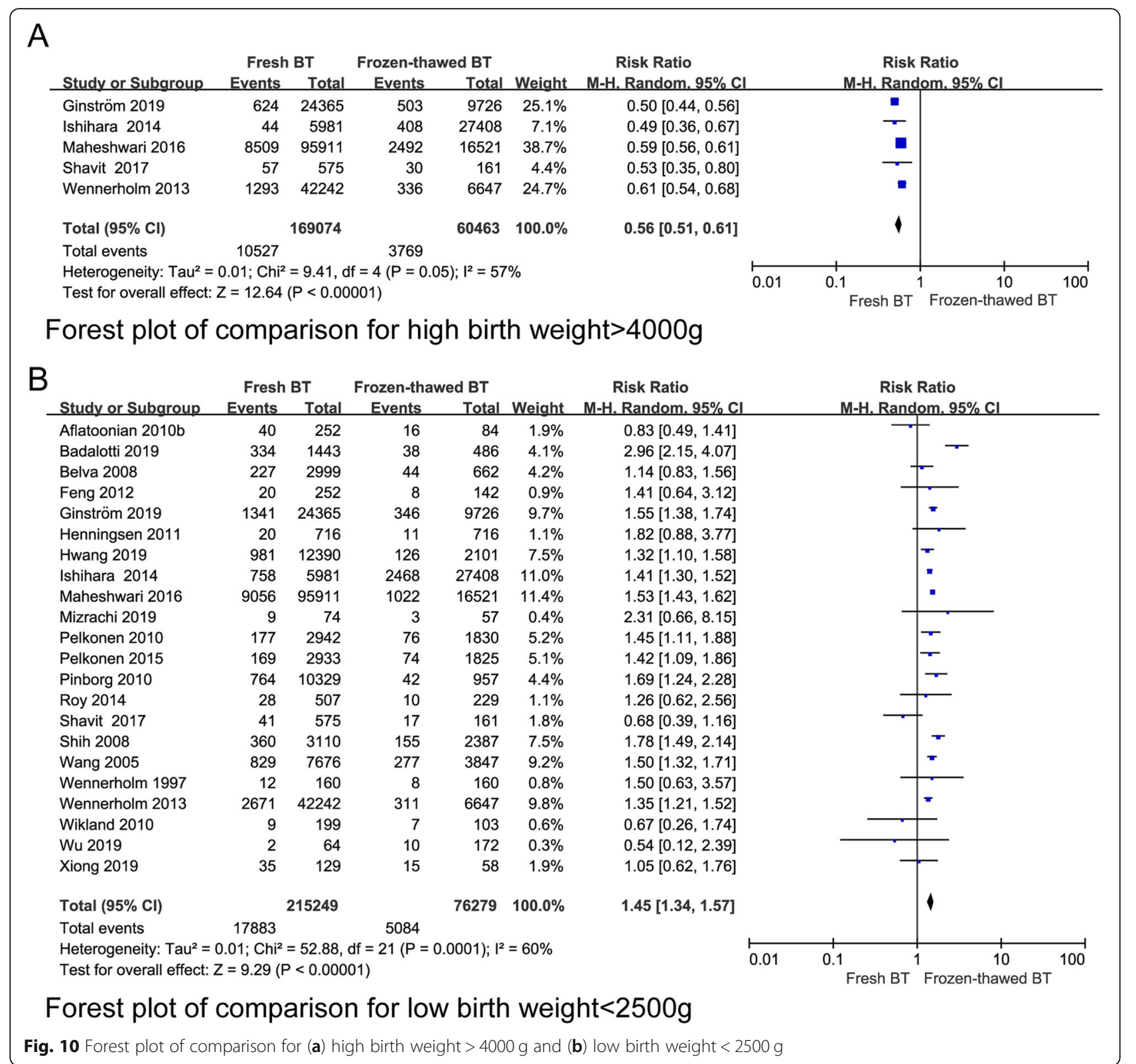

after fresh BT, which was consistent with the previous meta-analysis [2]. However, the stillbirth and perinatal mortality and neonatal mortality rates were not significantly different between the two groups. Moreover, with respect to neonatal malformations, there were no differences between fresh BT and frozen-thawed BT. In conclusion, singleton pregnancies after frozen-thawed BT seem to have better neonatal outcomes than those after fresh BT, owing to lower risks of SGA and LBW. The reasons for the association of better neonatal outcomes with frozen BT than with fresh BT are not yet known. Evidence from a recent original study showed that uterine Doppler studies in pregnancies from frozen-thawed blastocysts present an approximately 15\% lower uterine artery pulsatility index than those from fresh blastocysts, with a lower risk of SGA in the fresh group than in the frozen group, which is consistent with our conclusion [20] . In contrast to IVF with fresh embryo transfer, FET is usually performed in minimally stimulated or natural cycles. This lowers the risk of SGA and LBW after FET, which may be attributed to a luteal phase that mirrors the natural cycle, with favourable effects on the endometrium and early implantation [72]. Another probable explanation is related to the finding that controlled ovarian hyperstimulation $(\mathrm{COH})$ was associated with poorer neonatal outcomes assessed by SGA and LBW in a recent study [76]. The results favouring frozen thawed BT instead of fresh BT may relate to the adverse effects of 


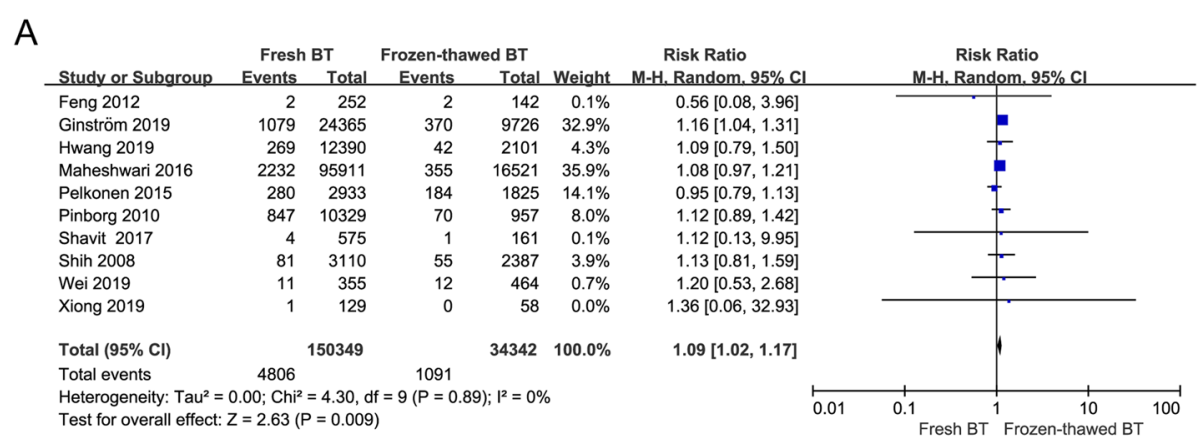

Forest plot of comparison for congenital anomaly and chromosomal aberrations

B

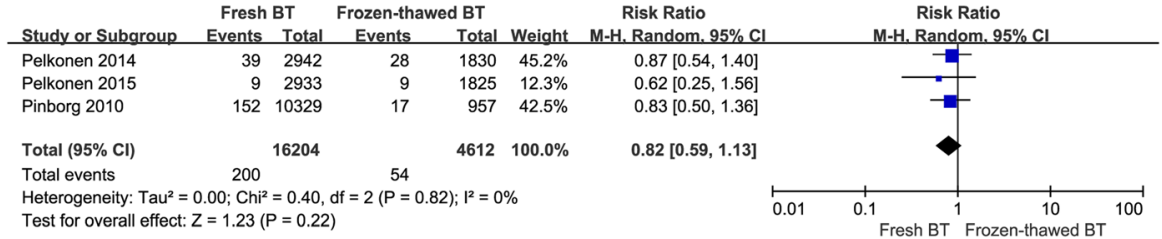

Forest plot of comparison for circulatory system diseases

C

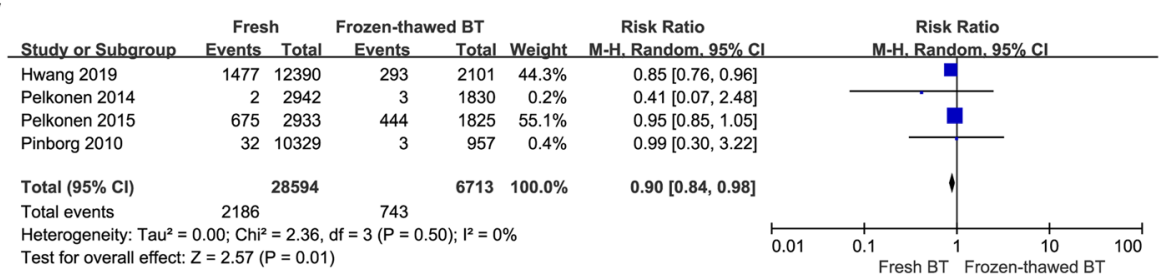

Forest plot of comparison for respiratory system diseases

D

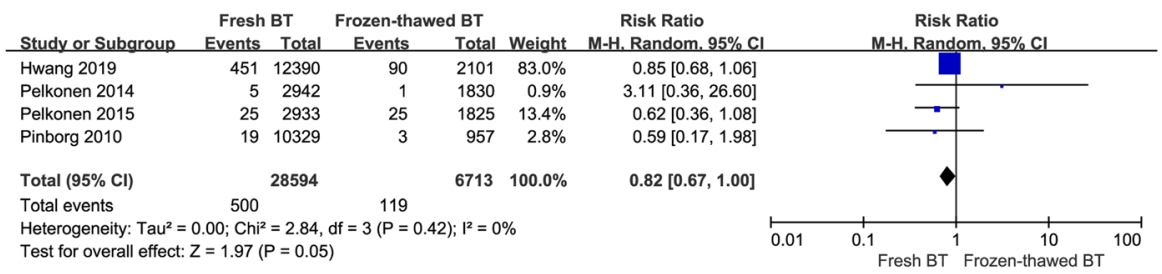

Forest plot of comparison for nervous system diseases.

Fig. 11 Forest plot of comparison for (a) congenital anomaly and chromosomal aberrations, (b) circulatory. System diseases, (c) respiratory system diseases and (d) nervous system diseases

$\mathrm{COH}$ on ER [48, 77]. Therefore, elective cryopreservation of viable embryos could be an alternative to avoid the deleterious effects of $\mathrm{COH}$ in embryo endometrium synchrony $[12,47]$.

\section{Strengths and limitations}

The major strength of this systematic review is the comprehensive literature search, identifying study subjects from a substantial number of relevant publications; another strength is the many pregnancy outcomes, maternal complications and neonatal outcomes the study evaluated between frozen and fresh BT to explore which method is better for mothers and infants. In addition, we conducted RCT and NRCT meta-analyses separately to improve the quality of evidence. However, the present meta-analysis also has some limitations, one of which was the significant heterogeneity in the pregnancy outcomes in the metaanalysis. We tried to find the source of heterogeneity by performing a subgroup analysis to examine the source of heterogeneity but were not able to identify the source. In addition, the baseline characteristics of patients differed among the included studies, including country, age, smoking status, duration of infertility, type of infertility, endometrial thickness, and cryopreservation type.

\section{Conclusions}

In summary, considering the higher IR, PR, OPR, and CPR; lower EPR; and decreased risks of OHSS, PA and 
PTD, as well as the incidences of SGA and LBW associated with frozen-thawed BT, there is an overall slight preponderance of risks in fresh cycles against frozen. However, frozen-thawed BT also has potential risks, such as LGA, PIH and pre-eclampsia. During the freezing process, there is a trade-off between the risk of preeclampsia hypertension and all other higher risks of fresh tranfers. In conclusion, the present review assessed the risks and magnitudes associated with fresh or frozen-thawed transfers, however it could not provide a final answer on the research question in object in the title. Therefore, individualization is required in order to choose the best procedure for each specific case according to the clinical needs.

\section{Supplementary information}

Supplementary information accompanies this paper at https://doi.org/10. 1186/s12884-020-03248-5.

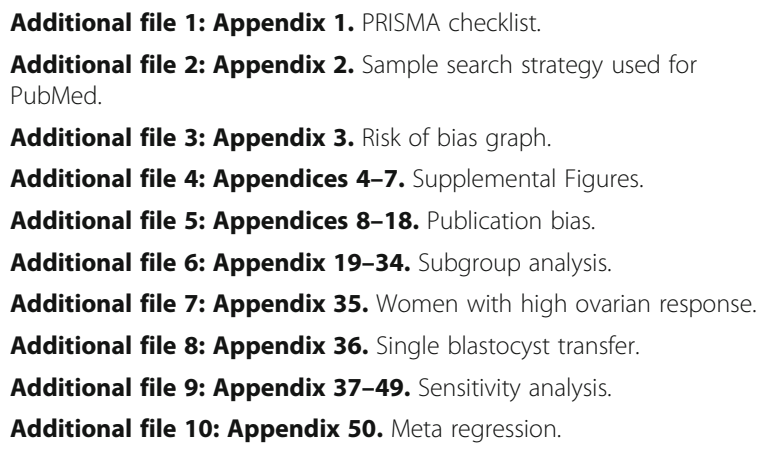

\section{Abbreviations}

BT: Blastocyst transfer; PH: Postpartum hemorrhage; PA: Placental abruption; PP: Placenta previa; GDM: Gestational diabetes mellitus; OHSS: Ovarian hyperstimulation syndrome; SGA: Small for gestational age; LBW: Low birth weight; PIH: Pregnancy-induced hypertension; LGA: Large for gestational age; VSGA: Very small for gestational age; HBW: High birth weight baby; VHBW: Very high birth weight baby (VHBW); RR: Risk ratio; Cl: Confidence intervals; RCT: Randomized controlled trials; NRCT: Non-randomized controlled trials; IR: Implantation rate; PR: Pregnancy rate; OPR: Ongoing pregnancy rate; EPR: Ectopic pregnancy rate; LBR: Live birth rate; MR: Miscarriage; MPR: Multiple pregnancy rate

\section{Acknowledgements}

Not applicable.

\section{Authors' contributions}

$X Z^{*}$ and $M Y$ conceived and designed the review. $X Z^{*}$ carried out activities from inception to the draft of the manuscript and is the guarantor of the review. $X Z^{*}$ and $M Y$ developed the search strings, selection, analysis and interpretation. XZ* , MY, LL, CS, TL, LC, WG, YM and YW rigorously review the manuscript. All authors read and approved the final version of the manuscript.

\section{Funding}

This study was funded by Social Development Project of Jiangsu (Grant number: BE2018693), Six talent peaks project in Jiangsu Province (Grant number:2016-WSW-125), Jiangsu Provincial Medical Youth Talent (Grant number: QNRC2016460), and Jiangsu Provincial maternal and child health young talents (Grant number: FRC201788). The fundings had no role in study design, data collection and analysis, decision to publish, or preparation of the manuscript.
Availability of data and materials

Not applicable.

Ethics approval and consent to participate

Not applicable.

\section{Consent for publication}

Not applicable.

\section{Competing interests}

The authors declare that they have no competing interests.

\section{Author details}

${ }^{1}$ Reproductive Medicine Center, The Fourth Affiliated Hospital of Jiangsu University (Zhenjiang Maternal and Child Health Hospital), 20 Zhengdong Road, Zhenjiang, Jiangsu 212001, People's Republic of China. ${ }^{2}$ Obstetrics and Gynecology, Nantong City No 1 People's Hospital and Second Affiliated Hospital of Nantong University, Nantong 226000, China. ${ }^{3}$ Reproductive Sciences Institute, Jiangsu University, Zhenjiang 212001, Jiangsu, China.

Received: 4 March 2020 Accepted: 14 September 2020

Published online: 23 September 2020

\section{References}

1. Kuwayama M, Vajta G, Kato O, Leibo SP. Highly efficient vitrification method for cryopreservation of human oocytes. Reprod BioMed Online. 2005;11(3): 300-8.

2. Maheshwari A, Pandey S, Shetty A, Hamilton M, Bhattacharya S. Obstetric and perinatal outcomes in singleton pregnancies resulting from the transfer of frozen thawed versus fresh embryos generated through in vitro fertilization treatment: a systematic review and meta-analysis. Fertil Steril. 2012;98(2):368-77 e1-9.

3. Zeng M, Li L. Single fresh blastocyst transfer or single cryopreserved-thawed blastocyst transfer: which is preferable for infertile patients in IVF/ICSI cycles? a meta analysis. Gynecol Endocrinol. 2019;35(1):17-22.

4. Roque M, Lattes K, Serra S, Sola I, Geber S, Carreras R, Checa MA. Fresh embryo transfer versus frozen embryo transfer in in vitro fertilization cycles: a systematic review and meta-analysis. Fertil Steril. 2013;99(1):156-62.

5. Dieamant FC, Petersen CG, Mauri AL, Comar V, Mattila M, Vagnini LD, Renzi A, Petersen B, Nicoletti A, Oliveira JBA, et al. Fresh embryos versus freeze-all embryos - transfer strategies: nuances of a meta-analysis. JBRA Assist Reprod. 2017;21(3):260-72.

6. Shavit T, Oron G, Weon-Young S, Holzer H, Tulandi T. Vitrified-warmed single-embryo transfers may be associated with increased maternal complications compared with fresh single-embryo transfers. Reprod BioMed Online. 2017;35(1):94-102.

7. Wei D, Liu J-Y, Sun Y, Shi Y, Zhang B, Liu J-Q, Tan J, Liang X, Cao Y, Wang Z, et al. Frozen versus fresh single blastocyst transfer in ovulatory women: a multicentre, randomised controlled trial. Lancet. 2019;393(10178):1310-8.

8. McDonald S, Murphy K, Beyene J, Ohlsson A. Perinatal outcomes of in vitro fertilization twins: a systematic review and meta-analyses. Am J Obstet Gynecol. 2005;193(1):141-52

9. Pelkonen S, Hartikainen AL, Ritvanen A, Koivunen R, Martikainen H, Gissler $M$, Tiitinen A. Major congenital anomalies in children born after frozen embryo transfer: a cohort study 1995-2006. Hum Reprod. 2014;29(7):1552-7.

10. Higgins JP, Altman DG, Gøtzsche PC, Jüni P, Moher D, Oxman AD, Savovic J, Schulz KF, Weeks L, Sterne JA. The Cochrane Collaboration's tool for assessing risk of bias in randomised trials. BMJ. 2011;343:d5928.

11. Higgins J. PT. measuring inconsistency in meta-analyses. BMJ. 2003; 327(7414):557-60.

12. Aflatoonian A, Oskouian H, Ahmadi S, Oskouian L. Can fresh embryo transfers be replaced by cryopreserved-thawed embryo transfers in assisted reproductive cycles? A randomized controlled trial. J Assist Reprod Genet. 2010;27(7):357-63.

13. Aflatoonian A, Mansoori Moghaddam F, Mashayekhy M, Mohamadian F. Comparison of early pregnancy and neonatal outcomes after frozen and fresh embryo transfer in ART cycles. J Assist Reprod Genet. 2010;27(12):695700.

14. Aghahosseini M, Aleyasin A, Sarfjoo FS, Mahdavi A, Yaraghi M, Saeedabadi $H$. In vitro fertilization outcome in frozen versus fresh embryo transfer in 
women with elevated progesterone level on the day of HCG injection: an RCT. Int J Reprod Biomed. 2017;15(12):757-62.

15. Ainsworth AJ, Wyatt MA, Shenoy CC, Hathcock M, Coddington CC. Fresh versus frozen embryo transfer has no effect on childhood weight. Fertil Steril. 2019;112(4):684-90 e1.

16. Badalotti M, Flach $\mathrm{S}$, Hentschke MR, Wingert F, Azambuja R, Trindade VD, Okada L, Tagliani-Ribeiro A, Proença LA, Cunegatto B, et al. Perinatal outcomes after fresh versus frozen-thawed embryo transfers. Fertil Steril. 2019;112(3):e298.

17. Beltran Anzola A, Pauly V, Riviere O, Sambuc R, Boyer P, Vendittelli F, Gervoise-Boyer MJ. Birthweight of IVF children is still a current issue and still related to maternal factors. Reprod BioMed Online. 2019;39(6):990-9.

18. Belva F, Henriet S, Van den Abbeel E, Camus M, Devroey P, Van der Elst J, Liebaers I, Haentjens P, Bonduelle M. Neonatal outcome of 937 children born after transfer of cryopreserved embryos obtained by ICSI and IVF and comparison with outcome data of fresh ICSI and IVF cycles. Hum Reprod. 2008;23(10):2227-38

19. Bourdon M, Santulli P, Maignien C, Gayet V, Pocate-Cheriet K, Marcellin L, Chapron C. The deferred embryo transfer strategy improves cumulative pregnancy rates in endometriosis-related infertility: a retrospective matched cohort study. PLoS One. 2018;13(4)::0194800.

20. Cavoretto P, Farina A, Gaeta G, Sigismondi C, Spinillo S, Casiero D, Pozzoni M, Vigano P, Papaleo E, Candiani M. Longitudinal cohort study of uterine artery Doppler in singleton pregnancies obtained by IVF/ICSI with fresh or frozen blastocyst transfers in relation to pregnancy outcomes. Ultrasound Obstet Gynecol. 2020;76(3):219-69.

21. Chen ZJ, Shi Y, Sun Y, Zhang B, Liang X, Cao Y, Yang J, Liu J, Wei D, Weng $\mathrm{N}$, et al. Fresh versus frozen embryos for infertility in the polycystic ovary syndrome. N Engl J Med. 2016;375(6):523-33.

22. Coates A, Kung A, Mounts E, Hesla J, Bankowski B, Barbieri E, Ata B, Cohen J, Munne S. Optimal euploid embryo transfer strategy, fresh versus frozen, after preimplantation genetic screening with next generation sequencing: a randomized controlled trial. Fertil Steril. 2017;107(3):723-30 e3.

23. Eum JH, Park JK, Kim SY, Paek SK, Seok HH, Chang EM, Lee DR, Lee WS. Clinical outcomes of single versus double blastocyst transfer in fresh and vitrified-warmed cycles. Clin Exp Reprod Med. 2016;43(3):164-8.

24. Fauque P, Jouannet P, Davy C, Guibert J, Viallon V, Epelboin S, Kunstmann $\mathrm{JM}$, Patrat C. Cumulative results including obstetrical and neonatal outcome of fresh and frozen-thawed cycles in elective single versus double fresh embryo transfers. Fertil Steril. 2010;94(3):927-35.

25. Feng G, Zhang B, Zhou H, Shu J, Gan X, Wu F, Deng X. Comparable clinical outcomes and live births after single vitrified-warmed and fresh blastocyst transfer. Reprod BioMed Online. 2012;25(5):466-73.

26. Ferraretti AP, Gianaroli L, Magli C, Fortini D, Selman HA, Feliciani E. Elective cryopreservation of all pronucleate embryos in women at risk of ovarian hyperstimulation syndrome: efficiency and safety. Hum Reprod. 1999;14(6): 1457-60.

27. Ginstrom Ernstad E, Wennerholm UB, Khatibi A, Petzold M, Bergh C. Neonatal and maternal outcome after frozen embryo transfer: Increased risks in programmed cycles. Am J Obstet Gynecol. 2019;221(2):126 e1-e18.

28. Healy DL, Breheny S, Halliday J, Jaques A, Rushford D, Garrett C, Talbot JM Baker HW. Prevalence and risk factors for obstetric haemorrhage in 6730 singleton births after assisted reproductive technology in Victoria Australia. Hum Reprod. 2010;25(1):265-74.

29. Henningsen AK, Pinborg A, Lidegaard O, Vestergaard C, Forman JL, Andersen AN. Perinatal outcome of singleton siblings born after assisted reproductive technology and spontaneous conception: Danish national sibling-cohort study. Fertil Steril. 2011;95(3):959-63.

30. Henman M, Catt JW, Wood T, Bowman MC, de Boer KA, Jansen RP. Elective transfer of single fresh blastocysts and later transfer of cryostored blastocysts reduces the twin pregnancy rate and can improve the in vitro fertilization live birth rate in younger women. Fertil Steril. 2005;84(6):1620-7.

31. Hwang SS, Dukhovny D, Gopal D, Cabral H, Diop H, Coddington CC, Stern JE. Health outcomes for Massachusetts infants after fresh versus frozen embryo transfer. Fertil Steril. 2019;112(5):900-7.

32. Ishihara O, Araki R, Kuwahara A, Itakura A, Saito H, Adamson GD. Impact of frozen-thawed single-blastocyst transfer on maternal and neonatal outcome: an analysis of 277,042 single-embryo transfer cycles from 2008 to 2010 in Japan. Fertil Steril. 2014;101(1):128-33.
33. Johnson KM, Hacker MR, Resetkova N, O'Brien B, Modest AM. Risk of ischemic placental disease in fresh and frozen embryo transfer cycles. Fertil Steril. 2019;111(4):714-21.

34. Korosec S, Virant-Klun I, Tomazevic T, Zech NH, Meden-Vrtovec H. Single fresh and frozen-thawed blastocyst transfer using hyaluronan-rich transfer medium. Reprod BioMed Online. 2007:15(6):701-7.

35. Le KD, Vuong LN, Ho TM, Dang VQ, Pham TD, Pham CT, Norman RJ, Mol BWJ. A cost-effectiveness analysis of freeze-only or fresh embryo transfer in IVF of non-PCOS women. Hum Reprod. 2018;33(10):1907-14.

36. Liu X, Bai H, Shi W, Shi J. Frozen-thawed embryo transfer is better than fresh embryo transfer in GnRH antagonist cycle in women with 3-10 oocytes retrieved: a retrospective cohort study. Arch Gynecol Obstet. 2019;300(6): $1791-6$.

37. Magdi Y, El-Damen A, Fathi AM, Abdelaziz AM, Abd-Elfatah Youssef M, AbdAllah AA, Ahmed Elawady M, Ahmed Ibrahim M, Edris Y. Revisiting the management of recurrent implantation failure through freeze-all policy. Fertil Steril. 2017;108(1):72-7.

38. Maheshwari A, Raja EA, Bhattacharya S. Obstetric and perinatal outcomes after either fresh or thawed frozen embryo transfer: an analysis of 112,432 singleton pregnancies recorded in the human fertilisation and embryology authority anonymized dataset. Fertil Steril. 2016;106(7):1703-8.

39. Martikainen $H$, Tiitinen A, Tomas $C$, Tapanainen J, Orava M, Tuomivaara L, Vilska S, Hyden-Granskog C, Hovatta O. One versus two embryo transfer after IVF and ICSI: a randomized study. Hum Reprod. 2001;16(9):1900-3.

40. Martikainen H, Orava M, Lakkakorpi J, Tuomivaara L. Day 2 elective single embryo transfer in clinical practice: better outcome in ICSI cycles. Hum Reprod. 2004;19(6):1364-6.

41. Mizrachi Y, Weissman A, Buchnik Fater G, Torem M, Horowitz E, Schreiber L, Raziel A, Bar J, Kovo M. Placental histopathology in IVF pregnancies resulting from the transfer of frozen-thawed embryos compared with fresh embryos. J Assist Reprod Genet. 2020;37(5):1155-62.

42. Pelkonen S, Koivunen R, Gissler M, Nuojua-Huttunen S, Suikkari AM, HydenGranskog C, Martikainen H, Tiitinen A, Hartikainen AL. Perinatal outcome of children born after frozen and fresh embryo transfer: the Finnish cohort study 1995-2006. Hum Reprod. 2010;25(4):914-23.

43. Pelkonen S, Gissler M, Koivurova S, Lehtinen S, Martikainen $\mathrm{H}$, Hartikainen AL, Tiitinen A. Physical health of singleton children born after frozen embryo transfer using slow freezing: a 3-year follow-up study. Hum Reprod. 2015;30(10):2411-8.

44. Pinborg A, Loft A, Aaris Henningsen AK, Rasmussen S, Andersen AN. Infant outcome of 957 singletons born after frozen embryo replacement: the Danish National Cohort Study 1995-2006. Fertil Steril. 2010;94(4):1320-7.

45. Roy TK, Bradley CK, Bowman MC, McArthur SJ. Single-embryo transfer of vitrified-warmed blastocysts yields equivalent live-birth rates and improved neonatal outcomes compared with fresh transfers. Fertil Steril. 2014;101(5): 1294-301.

46. Sik A, Oral S, Aba YA, Ozolcay O, Koc M, Sismanoglu A. Pregnancy results after fresh embryo transfer and selective frozen-thawed embryo transfer: single-center experience. J Gynecol Obstet Hum Reprod. 2020;49(4):101707.

47. Shapiro BS, Daneshmand ST, Garner FC, Aguirre M, Hudson C, Thomas S. Evidence of impaired endometrial receptivity after ovarian stimulation for in vitro fertilization: a prospective randomized trial comparing fresh and frozen-thawed embryo transfer in normal responders. Fertil Steril. 2011;96(2): 344-8.

48. Shapiro BS, Daneshmand ST, Garner FC, Aguirre M, Hudson C, Thomas S. Evidence of impaired endometrial receptivity after ovarian stimulation for in vitro fertilization: a prospective randomized trial comparing fresh and frozen-thawed embryo transfers in high responders. Fertil Steril. 2011;96(2): 516-8.

49. Shapiro BS, Daneshmand ST, Restrepo H, Garner FC, Aguirre M, Hudson C. Matched-cohort comparison of single-embryo transfers in fresh and frozenthawed embryo transfer cycles. Fertil Steril. 2013;99(2):389-92.

50. Shih W, Rushford DD, Bourne H, Garrett C, McBain JC, Healy DL, Baker HW Factors affecting low birthweight after assisted reproduction technology: difference between transfer of fresh and cryopreserved embryos suggests an adverse effect of oocyte collection. Hum Reprod. 2008;23(7):1644-53.

51. Vuong $L T$, Dang VQ, Ho TM, Huynh BG, Ha DT, Pham TD, Nguyen LK, Norman RJ, Mol BW. Freeze-all versus fresh embryo transfer in IVF/ICSI, a randomised controlled trial (NCT02471573). Fertil Steril. 2016;106(3):e376. 
52. Vuong LN, Dang VQ, Ho TM, Huynh BG, Ha DT, Pham TD, Nguyen LK, Norman RJ, Mol BW. IVF transfer of fresh or frozen embryos in women without polycystic ovaries. N Engl J Med. 2018;378(2):137-47.

53. Walls ML, Hunter T, Ryan JP, Keelan JA, Nathan E, Hart RJ. In vitro maturation as an alternative to standard in vitro fertilization for patients diagnosed with polycystic ovaries: a comparative analysis of fresh, frozen and cumulative cycle outcomes. Hum Reprod. 2015;30(1):88-96.

54. Wang YA, Sullivan EA, Black D, Dean J, Bryant J, Chapman M. Preterm birth and low birth weight after assisted reproductive technology-related pregnancy in Australia between 1996 and 2000. Fertil Steril. 2005;83(6): 1650-8.

55. Wennerholm UB, Hamberger L, Nilsson L, Wennergren M, Wikland M, Bergh C. Obstetric and perinatal outcome of children conceived from cryopreserved embryos. Hum Reprod. 1997;12(8):1819-25.

56. Wennerholm UB, Henningsen AK, Romundstad LB, Bergh C, Pinborg A, Skjaerven R, Forman J, Gissler M, Nygren KG, Tiitinen A. Perinatal outcomes of children born after frozen-thawed embryo transfer: a Nordic cohort study from the CoNARTaS group. Hum Reprod. 2013;28(9):2545-53.

57. Wikland M, Hardarson T, Hillensjo T, Westin C, Westlander G, Wood M, Wennerholm UB. Obstetric outcomes after transfer of vitrified blastocysts. Hum Reprod. 2010;25(7):1699-707.

58. Wu K, Zhao H, Liu H, Li M, Ma S, Li C, Liu C, Chen ZJ. Day 3 ET, single blastocyst transfer (SBT) or frozen-thawed embryo transfer (FET): which is preferable for high responder patients in IVF/ICSI cycles? J Assist Reprod Genet. 2014;31(3):275-8.

59. Wu J, Yang $X$, Huang J, Kuang $Y$, Wang Y. Fertility and neonatal outcomes of freeze-all vs. fresh embryo transfer in women with advanced endometriosis. Front Endocrinol (Lausanne). 2019;10(3):770.

60. Xiong F, Li G, Sun Q, Wang S, Wan C, Chen P, Yao Z, Zhong H, Zeng Y. Clinical outcomes after transfer of blastocysts derived from frozen-thawed cleavage embryos: a retrospective propensity-matched cohort study. Arch Gynecol Obstet. 2019;300(3):751-61.

61. Yang S, Pang T, Li R, Yang R, Zhen X, Chen X, Wang H, Ma C, Liu P, Qiao J. The individualized choice of embryo transfer timing for patients with elevated serum progesterone level on the HCG day in IVF/ICSI cycles: a prospective randomized clinical study. Gynecol Endocrinol. 2015;31(5):355-8.

62. Zhang B, Wei D, Legro RS, Shi Y, Li J, Zhang L, Hong Y, Sun G, Zhang T, Li $W$, et al. Obstetric complications after frozen versus fresh embryo transfer in women with polycystic ovary syndrome: results from a randomized trial. Fertil Steril. 2018;109(2):324-9.

63. Trounson A, Mohr L. Human pregnancy following cryopreservation, thawing and transfer of an eight-cell embryo. Nature. 1983;305(5936):707-9.

64. Kansal Kalra S, Ratcliffe SJ, Milman L, Gracia CR, Coutifaris C, Barnhart KT. Perinatal morbidity after in vitro fertilization is lower with frozen embryo transfer. Fertil Steril. 2011:95(2):548-53.

65. Achache $\mathrm{H}$, Revel A. Endometrial receptivity markers, the journey to successful embryo implantation. Hum Reprod Update. 2006;12(6):731-46.

66. Pyrgiotis E, Sultan KM, Neal GS, Liu HC, Grifo JA, Rosenwaks Z. Ectopic pregnancies after in vitro fertilization and embryo transfer. J Assist Reprod Genet. 1994;11(2):79-84.

67. Huang B, Hu D, Qian K, Ai J, Li Y, Jin L, Zhu G, Zhang H. Is frozen embryo transfer cycle associated with a significantly lower incidence of ectopic pregnancy? An analysis of more than 30,000 cycles. Fertil Steril. 2014;102(5): 1345-9.

68. Shapiro BS, Daneshmand ST, De Leon L, Garner FC, Aguirre M, Hudson C. Frozen-thawed embryo transfer is associated with a significantly reduced incidence of ectopic pregnancy. Fertil Steril. 2012;98(6):1490-4.

69. Blazquez A, Garcia D, Vassena R, Figueras F, Rodriguez A. Risk of preeclampsia after fresh or frozen embryo transfer in patients undergoing oocyte donation. Eur J Obstet Gynecol Reprod Biol. 2018;227:27-31.

70. D'Angelo A, Amso NN. Embryo freezing for preventing ovarian hyperstimulation syndrome: a Cochrane review. Hum Reprod. 2002;17(11): 2787-94.

71. Wennerholm UB, Soderstrom-Anttila V, Bergh C, Aittomaki K, Hazekamp J, Nygren KG, Selbing A, Loft A. Children born after cryopreservation of embryos or oocytes: a systematic review of outcome data. Hum Reprod. 2009;24(9):2158-72.

72. Pinborg A, Wennerholm UB, Romundstad LB, Loft A, Aittomaki K Soderstrom-Anttila V, Nygren KG, Hazekamp J, Bergh C. Why do singletons conceived after assisted reproduction technology have adverse perinatal outcome? Systematic review and meta-analysis. Hum Reprod Update. 2013; 19(2):87-104.

73. Maheshwari A, Pandey S, Amalraj Raja E, Shetty A, Hamilton M, Bhattacharya S. Is frozen embryo transfer better for mothers and babies? Can cumulative meta-analysis provide a definitive answer? Hum Reprod Update. 2018;24(1): 35-58.

74. Cavoretto P, Candiani M, Giorgione V, Inversetti A, Abu-Saba MM, Tiberio F, Sigismondi C, Farina A. Risk of spontaneous preterm birth in singleton pregnancies conceived after IVF/ICSI treatment: meta-analysis of cohort studies. Ultrasound Obstet Gynecol. 2018;51(1):43-53.

75. Cavoretto PI, Giorgione V, Sotiriadis A, Viganò P, Papaleo E, Galdini A, Gaeta $\mathrm{G}$, Candiani M. IVF/ICSI treatment and the risk of iatrogenic preterm birth in singleton pregnancies: systematic review and meta-analysis of cohort studies. J Matern Fetal Neonatal Med. 2020;17(4):1-10.

76. Chung K, Coutifaris C, Chalian R, Lin K, Ratcliffe SJ, Castelbaum AJ, Freedman $M F$, Barnhart KT. Factors influencing adverse perinatal outcomes in pregnancies achieved through use of in vitro fertilization. Fertil Steril. 2006; 86(6):1634-41.

77. Zhu D, Zhang J, Cao S, Zhang J, Heng BC, Huang M, Ling X, Duan T, Tong GQ. Vitrified-warmed blastocyst transfer cycles yield higher pregnancy and implantation rates compared with fresh blastocyst transfer cycles--time for a new embryo transfer strategy? Fertil Steril. 2011:95(5):1691-5.

\section{Publisher's Note}

Springer Nature remains neutral with regard to jurisdictional claims in published maps and institutional affiliations.
Ready to submit your research? Choose BMC and benefit from:

- fast, convenient online submission

- thorough peer review by experienced researchers in your field

- rapid publication on acceptance

- support for research data, including large and complex data types

- gold Open Access which fosters wider collaboration and increased citations

- maximum visibility for your research: over $100 \mathrm{M}$ website views per year

At BMC, research is always in progress.

Learn more biomedcentral.com/submissions 